\title{
Les stèles funéraires musulmanes dites batu Aceh de l'État de
} Johor (Malaisie)

\section{Daniel Perret}

\section{Citer ce document / Cite this document :}

Perret Daniel. Les stèles funéraires musulmanes dites batu Aceh de l'État de Johor (Malaisie). In: Bulletin de l'Ecole française d'Extrême-Orient. Tome 87 №2, 2000. pp. 579-607;

doi : https://doi.org/10.3406/befeo.2000.3492

https://www.persee.fr/doc/befeo_0336-1519_2000_num_87_2_3492

Fichier pdf généré le 07/05/2018 


\begin{abstract}
Daniel Perret

Muslim graves with batu Aceh tombstones from Johor (Malaysia)

211 Muslim graves with batu Aceh tombstones which can be safely dated between the mid-15th c. and the beginning of the 18th c have been described during surveys conducted between 1996 and 1999 in the State of Johor (Malaysia) by the École française d'Extrême-Orient in co-operation with the Yayasan Warisan Johor. These graves are located on 36 sites in three areas: Johor river basin, Simpang Kiri river and Muar river basin. Detailed descriptions have enabled us to define 16 types of batu Aceh. Except a very few of them, the epitaphs bear no name and no date. Thus hypotheses regarding the precise dating and the identification of the graves can be based only on written sources, oral traditions and comparative analyses. Such hypotheses are suggested here for graves located in large graveyards in the vicinity of Kota Tinggi which make up more than half of all the graves with batu Aceh tombstones in Johor. The results show that questions such as chronology and diffusion of types as well as choice of type in connection with the status of the deceased could be precisely answered only with the help of more detailed descriptions of batu Aceh outside Johor that would enable a more comprehensive comparative analysis.
\end{abstract}

\title{
Résumé
}

Daniel Perret

Les stèles funéraires musulmanes dites batu Aceh de l'État de Johor (Malaisie)

Des prospections menées entre 1996 et 1999 dans l'État de Johor (Malaisie) par l'École française d'Extrême-Orient en coopération avec la Fondation pour l'histoire de Johor (Yayasan Warisan Johor) ont permis de recenser 211 tombes musulmanes à stèles batu Aceh que l'on peut dater entre le milieu du XVe siècle et le début du XVIIle siècle. Ces tombes sont présentes sur 36 sites regroupés en trois zones : le bassin de la rivière Johor, la rivière Simpang Kiri et le bassin de la rivière Muar. Ces stèles ont fait l'objet de descriptions précises permettant de définir 16 types présents à Johor. À de très rares exceptions près, les épitaphes ne comportent ni nom, ni date. Les hypothèses concernant la datation précise et l'identification de ces tombes reposent ainsi uniquement sur des sources écrites, des traditions orales et des analyses comparatives. De telles hypothèses sont proposées ici pour des sépultures des grands cimetières de Kota Tinggi qui représentent plus de la moitié du corpus. II s'avère que les problèmes de chronologie et de diffusion des différents types de batu Aceh ainsi que leur choix en rapport avec la position sociale du défunt ne pourront être éclaircis qu'après avoir enrichi le corpus à des fins comparatives en effectuant, pour toutes les autres batu Aceh du reste du monde malais, une description systématique similaire à celle qui vient d'être réalisée à Johor. 


\title{
Les stèles funéraires musulmanes dites batu Aceh de l'État de Johor (Malaisie)'
}

\author{
Daniel PERret
}

En Malaisie, comme dans le reste du monde malais occidental, les tombes musulmanes anciennes les plus nombreuses et les plus répandues ${ }^{2}$ ayant subsisté sont celles comportant des stèles en pierre dites batu Aceh (pierres d'Aceh) ${ }^{3}$. Ces stèles, qui présentent des matériaux, des formes et des décors caractéristiques, semblent être en effet le produit d'une tradition artistique qui a pris naissance avec la diffusion de l'islam au Nord de Sumatra à la fin du XIII ${ }^{\mathrm{e}}$ siècle ${ }^{4}$. Cette région de Sumatra en recèle en fait encore plusieurs milliers alors qu'on en compte quelques centaines seulement en péninsule Malaise, où la plus ancienne portant une date confirmée (sha'bân $857 \mathrm{H}$., août-septembre 1453) se trouve dans l'État de Johor, au sud de la péninsule Malaise. C'est justement dans l'État de Johor que plusieurs prospections menées entre 1996 et 1999 par l'École française d'Extrême-Orient en coopération avec la Fondation pour l'héritage de Johor (Yayasan Warisan Johor) ont permis de recenser 211 tombes à stèles batu Aceh que l'on peut

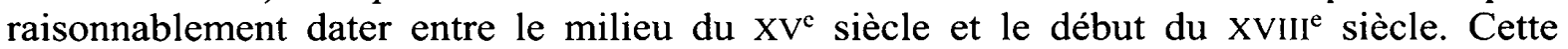
recherche constitue la première étape d'un programme d'inventaire des stèles batu Aceh du monde malais dont le but est d'en établir une typologie précise, de suivre la diffusion et de proposer une chronologie de ces types, d'essayer enfin d'identifier les tombes ne comportant pas d'inscriptions à caractère historique. Les principaux résultats de cette première étape sont présentés ici avec un essai de datation et d'identification des tombes des grands cimetières du secteur de Kota Tinggi.

1. Cet article est une synthèse partielle de l'ouvrage en malais paru en novembre 2000 intitulé Batu Aceh. Warisan Sejarah Johor, Johor Bahru, Yayasan Warisan Johor / EFEO, écrit en collaboration avec Kamarudin Ab. Razak et Ludvik Kalus (contribution épigraphique).

2. On en retrouve en effet du sud de la Thaïlande (région de Pattani) jusqu'aux Moluques et peutêtre même jusque dans l'archipel des Sulu aux Philippines.

3. Selon l'une des rares synthèses sur l'art funéraire musulman ancien en Indonésie, quatre grandes familles de stèles peuvent être distinguées : les stèles dites batu Aceh prédominantes à Sumatra et en péninsule Malaise, les stèles Demak-Troloyo prédominantes à Java, les stèles Bugis-Makassar prédominantes à Sulawesi et Sumbawa, enfin les stèles Ternate prédominantes dans l'archipel des Moluques. Ces quatre familles sont en fait le produit de quatre foyers principaux d'islamisation dans la région (Ambary, $1984: 341-363)$.

4. La tombe la plus ancienne portant une date confirmée est celle de Sultan Malik al-Salih, le premier sultan du royaume de Sumatra-Pasai. Elle est datée ramadan 696 H. (1297 E.C.). 


\section{Le contexte historique}

Dans l'histoire du monde malais, le nom de Johor est généralement associé au sultanat qui naît au début du $\mathrm{XVI}^{\mathrm{e}}$ siècle à l'extrémité méridionale de la péninsule Malaise. Successeur de Melaka comme grande puissance politique autochtone de la région, il va connaître son apogée au cours de la seconde moitié du XVII ${ }^{\mathrm{c}}$ siècle, profitant du déclin du sultanat d'Aceh et de la prise du Melaka portugais par les Hollandais en 1641.

Très peu de choses sont connues sur l'histoire de la région avant le $X V I^{\mathfrak{c}}$ siècle. On a cependant la preuve de l'existence d'habitats à Kampung Makam et à Johor Lama au XV siècle et au moins deux tombes à stèles batu Aceh sont datées avec certitude de la même époque. Il s'agit de la tombe de "la grand-mère du défunt seigneur Mansûr », dont l'épitaphe porte la date sha'bân 857 H. (août-septembre 1453) à Sayong Pinang ${ }^{5}$, et de la tombe du sultan Alauddin Riayat Syah I de Melaka à Kampung Raja (Pagoh), dont on sait par d'autres sources qu'il meurt en 1488.

Le sultanat de Johor naît dans l'urgence et se trouve menacé dès sa fondation. Pendant la décennie qui suit la prise de Melaka par les Portugais en 1511, le dernier sultan de Melaka, Mahmud Syah I, erre entre Johor (Muar, Pekan Tua ?, Pagoh), Pahang et l'île de Bintan d'où il sera chassé par les Portugais en 1526. Son successeur s'installe le long de la rivière Johor vers 1530 . Commence alors une longue série de déplacements de la capitale qui vont ponctuer toute l'histoire du sultanat. Entre 1535 et 1718 , on en compte en effet pas moins de vingt-deux le long du fleuve Johor dans la péninsule ainsi que dans l'archipel de Riau, mouvements qu'il faut mettre en partie au compte d'attaques portugaises et acihaises. La suite se déroulera dans l'archipel de Riau. C'est seulement en 1886 que Johor redeviendra capitale de sultanat avec l'installation de Maharaja Abu Bakar à Johor Bahru.

Onze sultans vont se succéder entre 1511 et 1718 : Sultan Mahmud Syah I (15111528), Sultan Alauddin Riayat Syah II (1528-1564), Sultan Muzzafar Syah II (1564-ca. 1569), Sultan Abdul Jalil I (ca. 1569), Sultan Abdul Jalil Syah II (ca. 1569-1597), Sultan Alauddin Riayat Syah III (1597-1613), Sultan Abdullah Ma'ayat Syah (1613-1623), Sultan Abdul Jalil III (1623-1677), Sultan Ibrahim I (1677-ca. 1685), Sultan Mahmud Syah II (ca. 1685-1699), Sultan Abdul Jalil IV (1699-1718).

Le développement du sultanat repose principalement sur sa capacité à attirer, quelquefois par la force, le trafic commercial maritime. La capitale est un carrefour d'échanges offrant une grande variété de marchandises ${ }^{6}$ avec des facilités telles que des entrepôts probablement enterrés pour éviter les incendies, des employés expérimentés pour l'organisation du commerce, des droits de douane concurrentiels, une stabilité des poids et mesures, la possibilité de réparer les navires sur place et l'absence de piraterie aux environs ${ }^{7}$. Chinois, Indiens (surtout Gujarati), Siamois, marchands du reste de la péninsule Malaise, Acihais et marchands de toute la côte de Sumatra, Javanais, marchands de Bornéo et de Célèbes, du Ténassirim, Anglais, Portugais ainsi que des Danois viennent

5. Ludvik Kalus in Perret et al., $1999: 127$.

6. $\mathrm{Au} \mathrm{XVII}{ }^{\mathrm{c}}$ siècle on peut acheter ou vendre à Johor de l'or de Jambi et d'Indragiri, de l'étain de Sumatra et d'une partie de la côte ouest de la péninsule Malaise, du cuivre japonais reçu de Patani par des marchands chinois, du sel du Siam, du bois d'aigle, du rotin, de la cire, du camphre, de l'ivoire, des nids d'hirondelles, du poivre de Jambi, Indragiri et Palembang, du riz de Java et du Siam, des soies, céramiques et quincaillerie chinoises, des vêtements d'Inde, du fil d'or japonais, de l'opium et des esclaves (Andaya, $1975: 38,69,76,128,148$ ).

7. Andaya, $1975: 38$. 
faire du commerce à Johor ${ }^{8}$. Les bateaux de Johor vont quant à eux jusqu'au golfe du Siam, au Bengale et au Coromandel ${ }^{9}$.

Au moment où les Hollandais prennent Melaka en 1641, Johor a autorité sur toute la côte ouest de la péninsule au sud de Selangor (sauf la région de Melaka), sur Siak, Kampar et Bengkalis à Sumatra, sur l'archipel de Riau-Lingga et les îles de Singapour. Progressivement, une bonne partie de la côte est de Sumatra au sud d'Aceh passe sous la domination de Johor vers la fin du XVII ${ }^{c}$ siècle ainsi que Terengganu, Pahang, la côte est de Johor, les îles Siantan et Tambelan ainsi que des îles proches de la côte ouest de Bornéo ${ }^{10}$.

\section{Traditions funéraires anciennes}

Les données épigraphiques des tombes à stèles batu Aceh de la péninsule Malaise indiquent que ces monuments étaient réservés aux sultans, aux grands personnages musulmans des sultanats ainsi qu'à leurs proches, dès le $X v^{e}$ siècle. Alors que les sources autochtones sont pratiquement muettes sur le sujet ${ }^{11}$, une source chinoise de la première moitié du XVI ${ }^{\mathrm{e}}$ siècle mentionne que les habitants de Melaka, qu'ils soient riches ou pauvres, brûlent leurs cadavres avec la seule différence que les cadavres des riches sont placés préalablement dans un cercueil avec du camphre ${ }^{12}$. Cette observation est confirmée à Johor par une autre source chinoise de la fin du XVI ${ }^{\mathrm{e}}$ siècle ou début du XVII ${ }^{\mathrm{e}}$ siècle qui mentionne que tous les cadavres sont brûlés ${ }^{13}$. Il semble qu'il faille attendre le XVII ${ }^{\mathrm{e}}$ siècle, voire le début du XVIII ${ }^{\mathrm{e}}$ siècle, pour que l'usage de la tombe se répande véritablement ${ }^{14}$, avec des stèles en galets, en pierres ordinaires ou en bois.

Deux sources écrites locales donnent en particulier des renseignements précis sur les pratiques funéraires concernant les hauts personnages. Le Bustan al-Salatin rédigé en 1640 par Nur-ud-din ar-Raniri contient trois faits intéressants à propos des stèles. Il apporte d'abord la preuve qu'au moins dès le début du XVII ${ }^{\mathrm{e}}$ siècle, des stèles de type batu Aceh sont envoyées d'Aceh vers la péninsule Malaise : il s'agit du passage relatant l'envoi par le sultan d'Aceh, Iskandar Thani, de stèles pour des tombes de Pahang ${ }^{15}$. Cette décision a des implications sur la datation des types de stèles. Il est clair en effet que ces stèles arrivent à Pahang plusieurs années (sinon dizaines d'années) après le décès et remplacent ${ }^{16}$ les stèles d'origine qui étaient peut-être également des batu Aceh. Ainsi, même pour des tombes de personnages dont la date de décès est connue (épitaphe ou

8. Ibid. : $25,69,71,76,104,128,147,148,149$.

9. Ibid. : $62,70,77$.

10. Ibid. : $37,148-149$.

11. L'article 3 des Undang-Undang Melaka mentionne seulement l'interdiction de l'usage du parasol et de la couleur jaune (Liaw Yock Fang éd., $1976: 68$ ).

12. Groeneveldt, $1880: 128$.

13. Il s'agit du T'ung Hsi Yang K'ao par Chang Hsieh, qui date de l'époque de l'empereur Wan Li (1573-1619). Cette source est reprise dans le Livre 325 de l'Histoire des Ming (Han Wai Toon, 1948: 31 ).

14. Le Hai-lu, une source chinoise de la fin du XVIII ${ }^{\mathrm{e}}$ siècle, ne mentionne plus de crémations mais la présence de tombes chez les Malais de la côte est de la péninsule Malaise (Cushman \& Milner, 1979: 13, 27).

15. Nur-ud-din ar-Raniri, Bustan al-Salatin, $1992: 40-42$. Linehan date cet épisode de 1638 et pense que le cimetière en question est celui de Makam Chondong près de la ville de Pekan. L'une des cinq tombes anciennes de ce cimetière serait celle de Puteri Bongsu Chendera Dewi, la propre mère d'Iskandar Thani (Linehan, 1973, [ $1^{\text {re }}$ éd. 1936] : 38-39, 235-236).

16. Dans au moins un manuscrit, c'est bien le terme menggantikan (remplacer) qui est employé (cf. Nuruddin al-Raniri, Bustan al-Salatin, 1992 : 41 n. 417 ; van Ronkel, 1920 : 170). 
autres sources), on ne peut être complètement certain que le type de batu Aceh qui marque ces tombes soit contemporain du décès. Une autre donnée intéressante concerne la décoration de certaines de ces stèles dont le même texte nous dit qu'elles sont recouvertes d'or et incrustées de pierres précieuses ${ }^{17}$. Si de telles stèles sont parvenues jusqu'à Johor, leurs décorations ont disparu; il faut donc considérer a priori les stèles batu Aceh visibles aujourd'hui comme incomplètes. Il en est de même des inscriptions de différentes couleurs peintes sur ces stèles, une pratique mentionnée dans un autre texte, l'Adat Raja-Raja Melayu, qui comprend un chapitre très intéressant sur les coutumes suivies à l'occasion du décès des sultans de Melaka ${ }^{18}$. Ce texte a été rédigé à Melaka par un certain Tuan Lebai Abdulmuhit en 1779 à la demande de De Bruyn, le Gouverneur hollandais de Melaka à l'époque ${ }^{19}$. Bien que collectées près de 300 ans après la chute du sultanat, ces informations apparaissent néanmoins vraisemblables, ceci d'autant plus que les autres sultanats de la péninsule Malaise reprendront une bonne partie de ces coutumes. Le texte apporte des détails sur les stèles elles-mêmes et en particulier sur leur fabrication par des artisans locaux ${ }^{20}$. Les descriptions montrent clairement que les stèles de type batu Aceh sont utilisées pour les tombes des sultans de Melaka. Il en subsiste d'ailleurs un exemple à Johor ${ }^{21}$ avec la tombe du sultan Alauddin Riayat Syah qui règne sur Melaka entre 1477 et 1488.

\section{Historique des recherches sur les batu Aceh de Johor}

La première étude sur les batu Aceh de Johor est publiée en 1932 par Richard Olaf Winstedt dans son long article "A History of Johore (A.D. 1365-1895) " ${ }^{22} .21$ sites avec un total de 137 tombes à batu Aceh y sont recensés. Dans son étude, Winstedt, conscient que ces stèles ont un lien avec Aceh, privilégie les données épigraphiques et limite la description des stèles à des expressions comme "Malayo-Achinese type ", " inferior specimens of the elaborately foliate type ", "European-lantern (or "pine-apple") type ", " usual XVIIth century type » ou encore "Chinese-lantern type ». La première édition de l'étude comporte d'ailleurs une bonne étude épigraphique d'un certain nombre de ces stèles par Engku 'Abdul'-Hamid bin Engku 'Abdu'l-Majid ${ }^{23}$. Le travail de Winstedt a un autre intérêt, celui de donner des informations précises sur la localisation des tombes.

Il faut attendre plus d'un demi-siècle pour voir apparaître la seconde étude importante concernant les batu Aceh de Johor. Il s'agit de la thèse de Othman bin Mohd. Yatim soutenue en 1985 et publiée en 1988 par le musée national de Malaisie. Elle repose sur des prospections menées entre 1974 et 1983 dans toute la péninsule Malaise et, contrairement au travail de Winstedt, a l'avantage de proposer une typologie précise basée sur la forme générale des batu Aceh de cette région. Les quatorze types de stèles batu Aceh définis par Othman bin Mohd. Yatim constituent encore la référence pour tous les travaux sur les batu Aceh et nous nous en sommes également inspirés pour établir la typologie des stèles de Johor. Du point de vue du nombre de stèles recensées à Johor, cette étude est néanmoins en recul par rapport à celle de Winstedt puisque Othman bin Mohd. Yatim ne mentionne

17. Nuruddin al-Raniri, Bustan al-Salatin, $1992: 45-46$.

18. Panuti H. M. Sudjiman (éd.), $1983: 92-95$.

19. Ibid. : 55-56.

20. Lors de nos prospections nous avons pu observer plusieurs stèles inachevées ou encore des tombes où l'une des deux stèles seulement a été travaillée, ce qui appuierait bien cette idée d'une fabrication locale à partir de pierres brutes qui, elles, proviendraient peut-être toutes du nord de Sumatra.

21. Kampung Raja, Pagoh, Muar, tombe KRAJ 1.

22. Une grande partie de cette étude a été réimprimée en 1979, puis en 1992.

23. Winstedt, 1932b : 159-167. 
que 83 tombes à batu $A c e h^{24}$ pour cet État. À la même époque, dans sa thèse sur les tombes musulmanes d'Indonésie (1984), Hasan Muarif Ambary propose une autre typologie des batu Aceh basée sur des stèles d'Indonésie et de Malaisie, y compris quelques exemplaires de Johor. En plus de l'étude épigraphique mentionnée plus haut, il faut aussi rappeler les contributions épigraphiques de Louis-Charles Damais (1968) et de Abdul Halim Nasir seul (1985) ou avec Othman bin Mohd. Yatim (1990).

Les prospections menées conjointement par l'École française d'Extrême-Orient et la Fondation pour l'héritage de Johor (Yayasan Warisan Johor) entre 1996 et 1999 ont recensé 211 tombes ${ }^{25}$ à batu Aceh dans 36 sites répartis sur trois grandes zones. Ce chiffre peut paraître faible si on le compare à la fourchette chronologique concernée, qui est d'un peu plus de deux siècles et demi. Il faut néanmoins avoir présent à l'esprit que ces tombes sont réservées à l'élite et qu'à Johor elles sont de très loin les plus nombreuses que l'on puisse attribuer avec suffisamment de certitude à cette période. A l'exception de quelques tombes en marbre rose plus ou moins sophistiquées relevant d'une autre tradition mais probablement contemporaines des batu Aceh, les nombreuses prospections effectuées confirment ainsi la très grande importance des stèles batu Aceh en tant que vestige pour la période et la région concernées. On peut sans grand risque étendre cette constatation à toute la péninsule Malaise ainsi qu'à Sumatra. Le nombre de stèles répertoriées s'est ainsi accru de 74 tombes (soit $54 \%$ ) par rapport à l'inventaire de Winstedt, qui était le plus complet jusqu'ici. La zone de loin la plus importante est le bassin de la rivière Johor, avec 195 tombes, suivie par la rivière Simpang Kiri et ses affluents dans la région de Batu Pahat avec dix tombes, et enfin la rivière Muar et ses affluents dans la région de Muar avec six tombes. Cette localisation des tombes à proximité des cours d'eau n'est pas pour surprendre dans la mesure où jusqu'au début du $\mathrm{XX}^{\mathrm{c}}$ siècle les cours d'eau ont constitué le moyen de communication principal dans la région. Un tableau de l'état de conservation des stèles permet d'observer qu'environ deux tiers des tombes ont encore au moins une stèle en bon état. Toutes les tombes repérées lors de ces prospections sont décrites précisément dans un catalogue par site avec les coordonnées topographiques obtenues par GPS et un plan succinct ${ }^{26}$. Ce catalogue est le premier du genre en ce qui concerne les batu Aceh.

La récente analyse par Ludvik Kalus des inscriptions du corpus de batu Aceh de Johor a permis de vérifier et parfois de modifier les lectures faites par Engku 'Abdul'-Hamid bin Engku 'Abdu'l-Majid ainsi que de proposer des lectures d'inscriptions inédites. Du point de vue épigraphique, les batu Aceh de Johor présentent, selon Ludvik Kalus ${ }^{27}$, deux groupes distincts : «Un premier groupe comprend les monuments funéraires contenant des textes lisibles, des épitaphes, même si ces dernières ne sont parfois constituées que de versets coraniques ou d'autres textes à caractère religieux. Le deuxième groupe comprend les monuments funéraires avec des inscriptions à lettres plus ou moins "stylisées", parfois totalement illisibles. "

24. Othman bin Mohd. Yatim, $1988: 102$.

25. Le véritable chiffre est toutefois difficile à connaître, car plusieurs tombes présentent deux stèles différentes du point de vue de la forme et des décors. Winstedt pense que dans ce cas il pourrait s'agir de deux tombes différentes (Winstedt, 1992 : 215). Il est clair par ailleurs que de nombreuses stèles ont été déplacées, notamment pour être installées dans des bâtiments commémoratifs, et que d'autres ont été arrachées. Ainsi la position actuelle des stèles ne constitue pas une preuve absolue pour rejeter l'hypothèse de Winstedt.

26. Perret et al., 1999.

27. Ludvik Kalus in Perret et al., 1999 : 104-105. 
Les sites à batu Aceh de l'État de Johor

\begin{tabular}{|c|c|c|c|c|}
\hline \multirow[t]{2}{*}{ Site } & \multirow[t]{2}{*}{ abrév. } & \multicolumn{3}{|c|}{ nombre de tombes } \\
\hline & & EFEO/YWJ & OTHMAN & WINSTEDT \\
\hline Ulu Sungai Che Omar & ULU & 24 & & 27 \\
\hline Bendahara Tun Habab & BEN & 22 & 23 & 23 \\
\hline Makam Tauhid & THD & 21 & 9 & 9 \\
\hline Sultan Mahmud & SMAH & 20 & 20 & 20 \\
\hline Kampung Makam & KMAK & 18 & 2 & 2 \\
\hline Makam 12 (Sayong Pinang) & SPDB & 11 & 11 & 11 \\
\hline Makam 7 (Sayong Pinang) & SPT & 8 & 8 & 10 \\
\hline Sayong Pinang & SAY & 7 & 6 & 4 \\
\hline Kampung Makam Dagang & KMD & 7 & & \\
\hline Batu Sawar & BSAW & 7 & & 5 \\
\hline Tanjung Belading & TBEL & 6 & & 4 \\
\hline Bukit Inas & INAS & 6 & & \\
\hline Bukit Seluyut & SELU & 5 & 5 & 5 \\
\hline Panchor & PAN & 5 & & 2 \\
\hline Danau Raja & DRAJ & 5 & & 4 \\
\hline Sungai Telor & STEL & 4 & & \\
\hline Sungai Tiram & STIR & 4 & & \\
\hline Kampung Lukut & LUK & 4 & & \\
\hline Pangkalan Rambai & PRAM & 3 & & \\
\hline Bukit Telepok & BT & 2 & & 2 \\
\hline Johor Lama & JOLA & 2 & 2 & 2 \\
\hline Kampung Sepak Hilir & SEPH & 2 & & \\
\hline Laksamana Bentan & LAK & 2 & & 1 \\
\hline Kampung Raja & KRAJ & 2 & 2 & 2 \\
\hline Lubok Batu & LUB & 2 & 1 & 2 \\
\hline Bukit Chapal & BCHA & 2 & & \\
\hline Sungai Cekedam & SCEK & 1 & & \\
\hline Makam Cekedam & CKD & 1 & & \\
\hline Panglima Dayong & PGD & 1 & & \\
\hline Sungai Pemandi & SPEM & 1 & & \\
\hline Siti Aminah & SIT & 1 & 1 & 1 \\
\hline Kota Buruk & KBUR & 1 & & 1 \\
\hline Kampung Berohol & KBER & 1 & 1 & \\
\hline Bukit Senjata & BSEN & 1 & & \\
\hline Bukit Kerayong & BKER & 1 & & \\
\hline Sungai Seluang & SSLG & 1 & & \\
\hline Pekan Tua & & & & fragments \\
\hline Kuala Sungai Johor & & & 11 & \\
\hline Total & & 211 & 102 & 137 \\
\hline
\end{tabular}




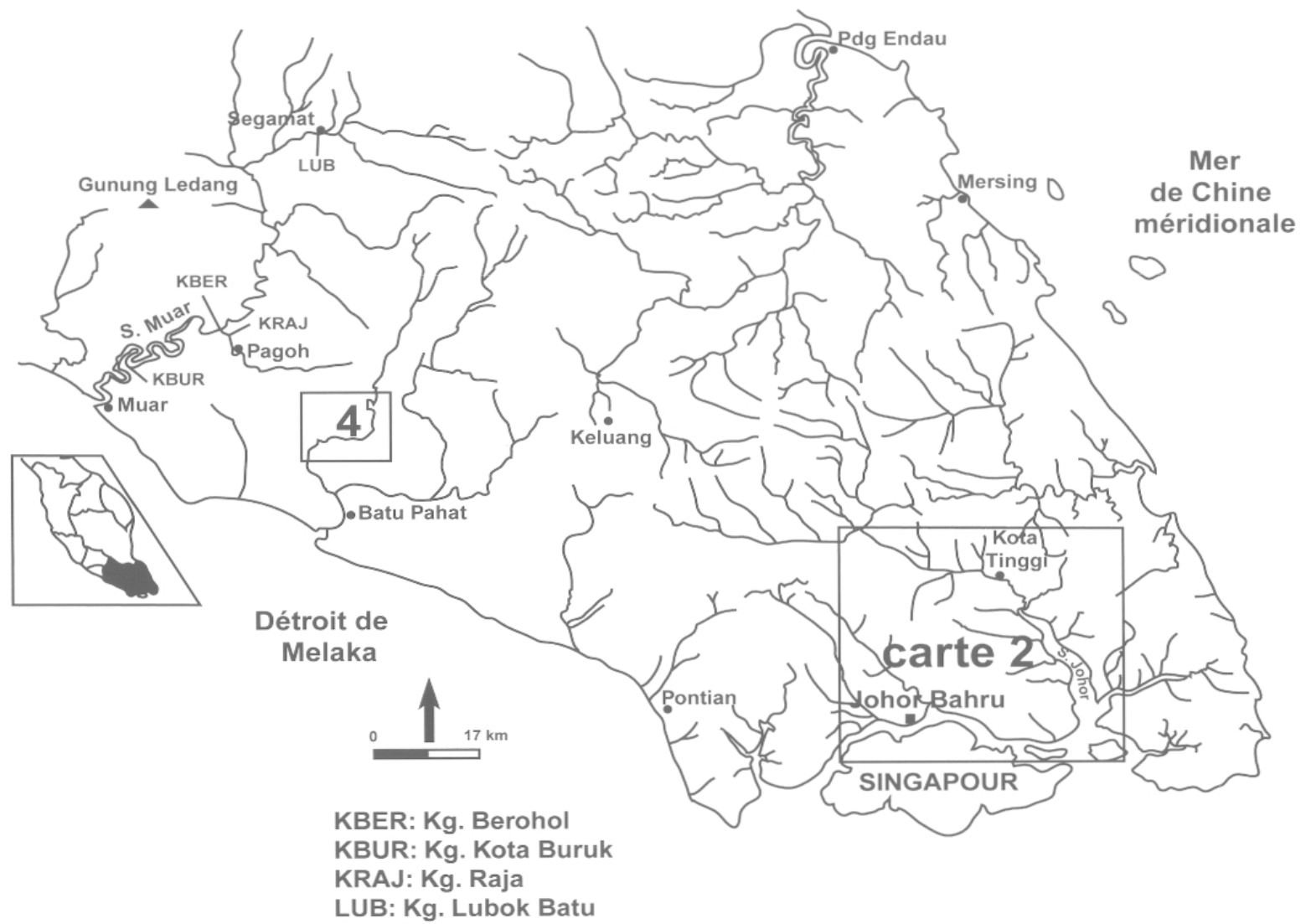

Carte 1. - Les trois zones à batu Aceh de l'État de Johor 


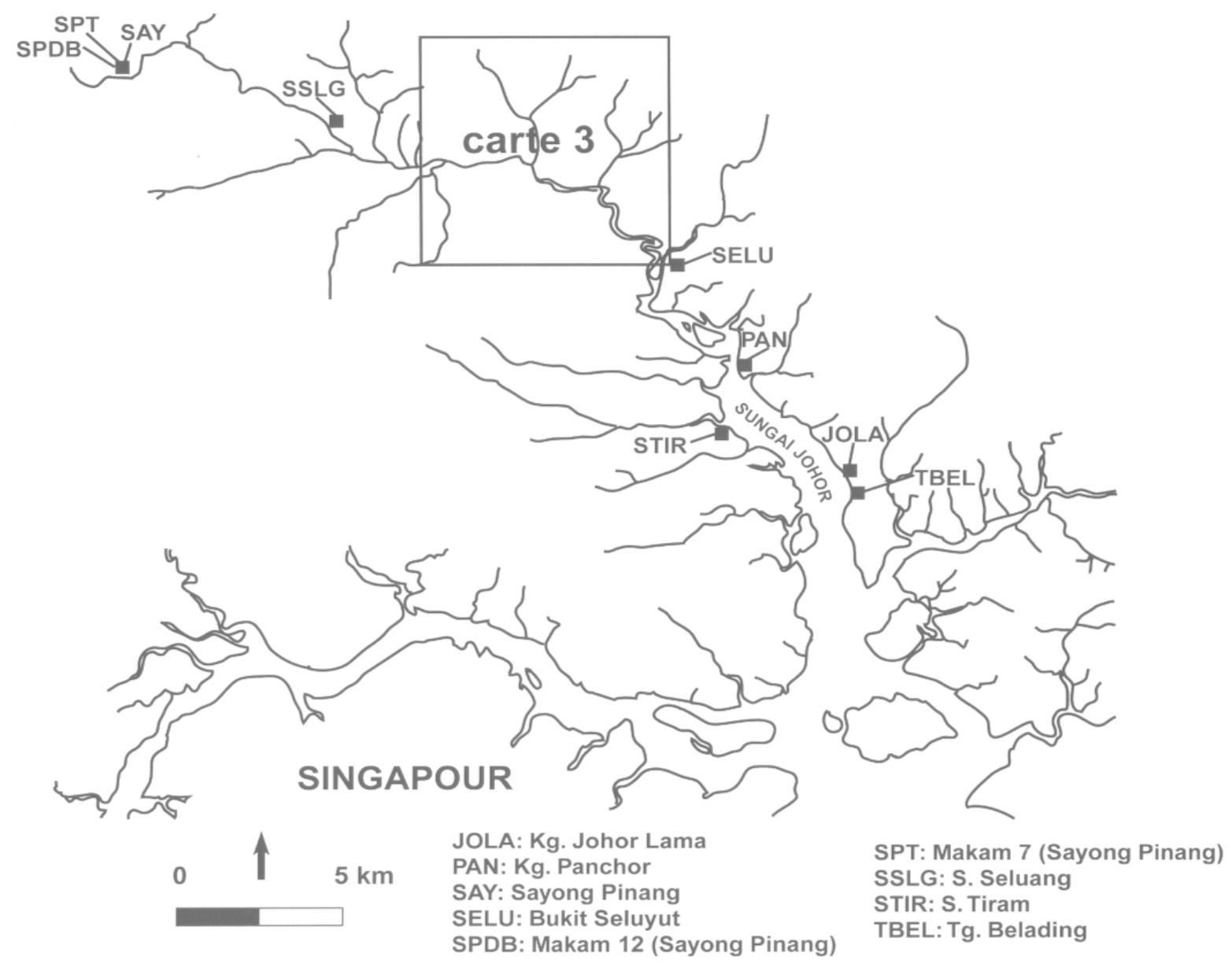

Carte 2. - Sites à batu Aceh du bassin de la rivière Johor 


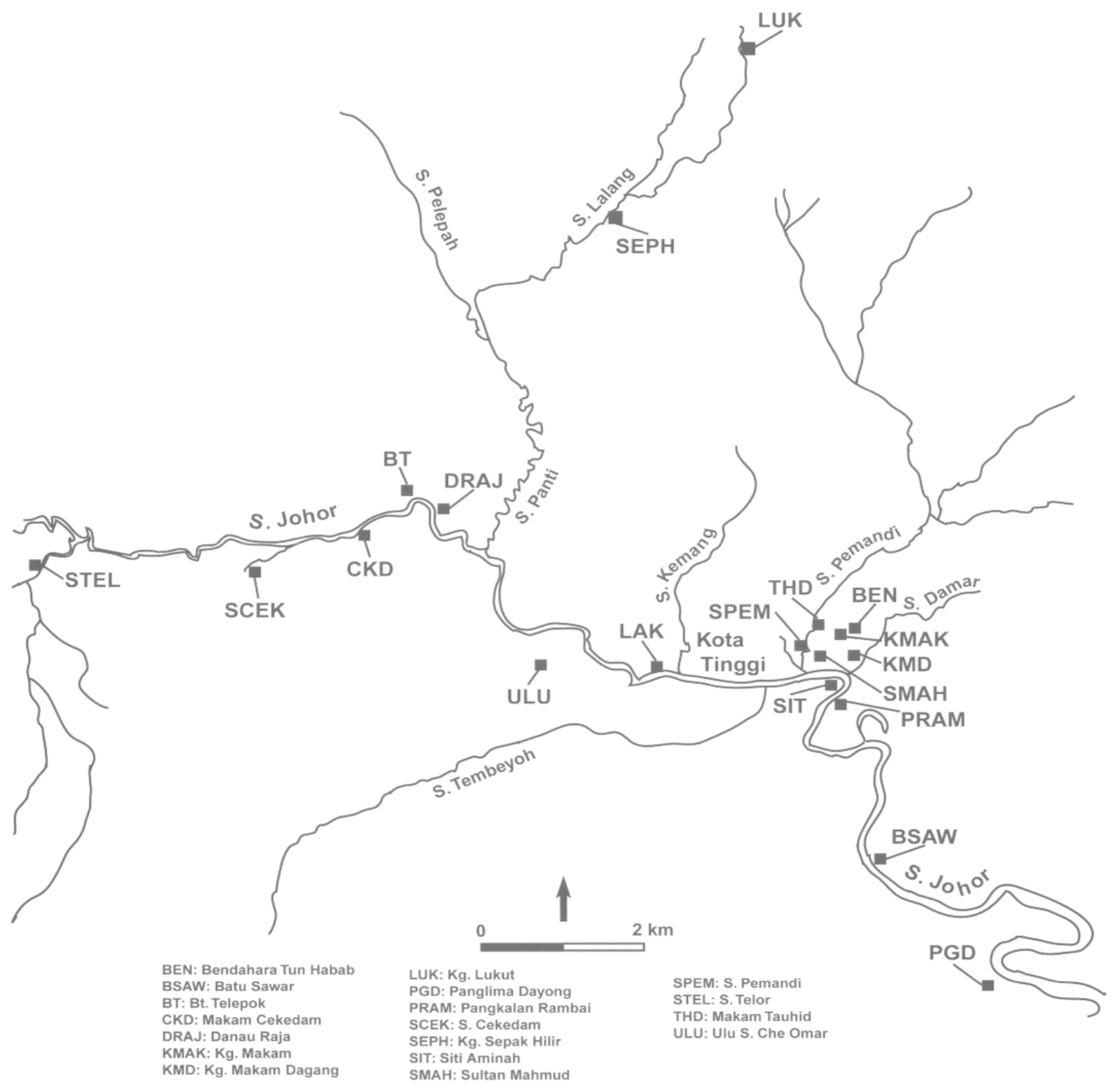

Carte 3. - Sites à batu Aceh du secteur de Kota Tinggi 


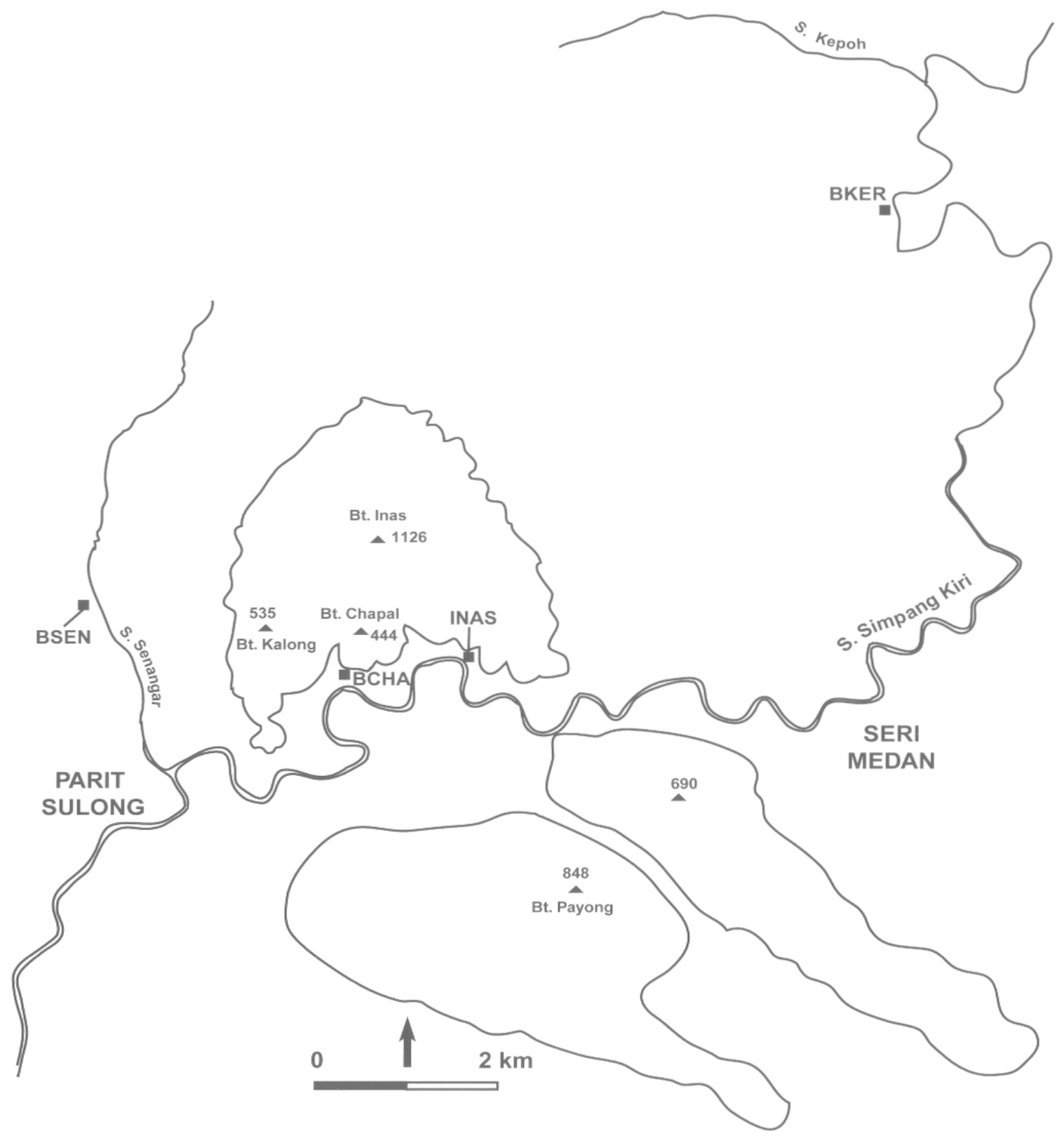

BCHA: Bt. Chapal BSEN: Bt. Senjata BKER: Bt. Kerayong INAS: Bt. Inas 
État de conservation des batu Aceh de Johor

\begin{tabular}{|c|c|c|c|c|c|}
\hline \multirow[t]{2}{*}{ Site } & \multicolumn{5}{|c|}{ État de la stèle la mieux conservée pour chaque tombe } \\
\hline & $90 \%-100 \%$ & $66 \%-90 \%$ & $25 \%-66 \%$ & $10 \%-25 \%$ & total \\
\hline Ulu Sungai Che Omar & 12 & 8 & 1 & 3 & 24 \\
\hline Bendahara Tun Habab & 15 & 6 & 1 & & 22 \\
\hline Makam Tauhid & 17 & 2 & & 2 & 21 \\
\hline Sultan Mahmud & 15 & 2 & 1 & 2 & 20 \\
\hline Kampung Makam & 13 & 2 & 1 & 2 & 18 \\
\hline Makam 12 (Sayong Pinang) & 11 & & & & 11 \\
\hline Makam 7 (Sayong Pinang) & 7 & 1 & & & 8 \\
\hline Sayong Pinang & 5 & 2 & & & 7 \\
\hline Kampung Makam Dagang & 4 & 2 & & 1 & 7 \\
\hline Batu Sawar & 2 & 3 & 2 & & 7 \\
\hline Tanjung Belading & 5 & 1 & & & 6 \\
\hline Bukit Inas & 6 & & & & 6 \\
\hline Bukit Seluyut & 3 & 1 & & 1 & 5 \\
\hline Panchor & 3 & 1 & & 1 & 5 \\
\hline Danau Raja & 1 & 2 & 1 & 2 & 5 \\
\hline Sungai Telor & 2 & 1 & 1 & & 4 \\
\hline Sungai Tiram & 1 & 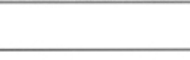 & & 3 & 4 \\
\hline Kampung Lukut & 1 & 1 & & 1 & 4 \\
\hline Pangkalan Rambai & 1 & 1 & 1 & & 3 \\
\hline Bukit Telepok & 2 & & & & 2 \\
\hline Johor Lama & 2 & & & & 2 \\
\hline Kampung Sepak Hilir & & 2 & & & 2 \\
\hline Laksamana Bentan & 2 & & & & 2 \\
\hline Kampung Raja & 2 & & & & 2 \\
\hline Lubok Batu & 2 & & & & 2 \\
\hline Bukit Chapal & 2 & & & & 2 \\
\hline Sungai Cekedam & & 1 & & & 1 \\
\hline Makam Cekedam & 1 & & & & 1 \\
\hline Panglima Dayong & 1 & & & & 1 \\
\hline Sungai Pemandi & & 1 & & & 1 \\
\hline Siti Aminah & 1 & & & & 1 \\
\hline Kota Buruk & 1 & & & & 1 \\
\hline Kampung Berohol & 1 & & & & 1 \\
\hline Bukit Senjata & 1 & & & & 1 \\
\hline Bukit Kerayong & 1 & & & & 1 \\
\hline Sungai Seluang & 1 & & & & 1 \\
\hline Total & 144 & 40 & 9 & 18 & 211 \\
\hline
\end{tabular}




\section{Typologie}

Seize types ont pu être identifiés d'après leur forme générale, dont neuf en forme de dalle (A, B, C, D, E, F, N, O, Q) et sept en forme de pilier (G, H, J, K, L, M, P) (cf. ill. p. 593). Le type le plus fréquent à Johor est $\mathrm{C}(42 \%)$, que l'on retrouve sur 26 sites, suivi de $\mathrm{K}(17 \%)$, A $(9 \%), \mathrm{H}(7 \%)$ et $\mathrm{O}(7 \%)$. Nos prospections renforcent ainsi la position du type $\mathrm{C}$ comme type prédominant en péninsule Malaise ${ }^{28}$. Treize de ces types ont été identifiés et repérés à Aceh par Othman bin Mohd. Yatim. Notre étude en ajoute trois : $O$, $P$ et $Q$. Une investigation préliminaire montre que le type $O$ est présent dans l'archipel de Riau, le type $\mathrm{P}$ à Aceh et le type $\mathrm{Q}$ dans la région de Pattani au sud de la Thaïlande. Trois tombes présentent des stèles dont le type ne peut être identifié avec certitude. Notre méthode d'analyse typologique reprend celle de Othman bin Mohd. Yatim, qui distingue six parties dans la description des stèles ( $c f$. illus. 2 pour le type $C$ ) : base (I), partie inférieure du corps (II), partie supérieure du corps (III), épaules (IV), tête (V) et sommet (VI). Les descriptions sont les plus précises jamais effectuées pour ce genre de stèles et puisent dans une liste de 252 détails d'ornementation relevés sur l'ensemble des stèles du corpus. Cette liste se veut la plus exhaustive possible, car dans l'état actuel des connaissances il est impossible de préjuger de la pertinence de telle ou telle caractéristique pour formuler des hypothèses de datation précise ou d'identification des tombes. Ces détails d'ornementation se répartissent de la façon suivante : base (57), partie inférieure du corps (22), partie supérieure du corps (61), épaules (30), tête (51) et sommet (31). Ils représentent des variantes pour un même type, mais peuvent également se retrouver sur plusieurs types. C'est ainsi que notre étude a permis d'observer que 112 d'entre eux sont communs à au moins deux types et que deux d'entre eux sont communs à huit types. Ces constatations contribuent à renforcer l'hypothèse selon laquelle ces stèles sont le produit d'une même tradition artistique. Le nombre de caractéristiques différentes illustre bien par ailleurs la variété et la richesse d'ornementation de ces stèles, qui sont probablement les plus sophistiquées dans l'histoire ancienne de la Malaisie.

Tous les sites comportant un minimum de deux tombes présentent au moins deux types de stèles, à l'exception de Bukit Telepok (BT) et de Kampung Raja (Pagoh) (KRAJ). Par ailleurs, chaque type représenté par au moins deux tombes se retrouve sur au moins deux sites. Ces constatations ont des implications importantes sur la chronologie des types. Le fait de définir un corpus de caractéristiques pour décrire les stèles permet d'établir des comparaisons entre les stèles et ainsi des regroupements basés sur le nombre de caractéristiques communes. Ces regroupements permettent à leur tour de proposer une datation, voire une identification du défunt si certaines caractéristiques communes sont très particulières. Nous présentons ci-dessous un exemple de regroupement où toutes les stèles d'un même type possèdent entre elles un minimum de huit et un maximum de quatorze caractéristiques communes.

\begin{tabular}{|c|c|c|c|c|c|c|c|}
\hline & DRAJ 1 & LAK 1 & ULU 23 & BEN 14 & THD 5 & BEN 17 & THD 4 \\
\hline LAK 1 & 14 & & & & & & \\
\hline ULU 23 & 13 & 13 & & & & & \\
\hline BEN 14 & 12 & 12 & 10 & & & & \\
\hline THD 5 & 12 & 11 & 12 & 13 & & & \\
\hline BEN 17 & 9 & 11 & 11 & 10 & 10 & & \\
\hline THD 4 & 10 & 10 & 10 & 11 & 12 & 9 & \\
\hline ULU 22 & 8 & 10 & 9 & 8 & 8 & 10 & 9 \\
\hline
\end{tabular}

28. Othman bin Mohd. Yatim, $1988: 46$. 

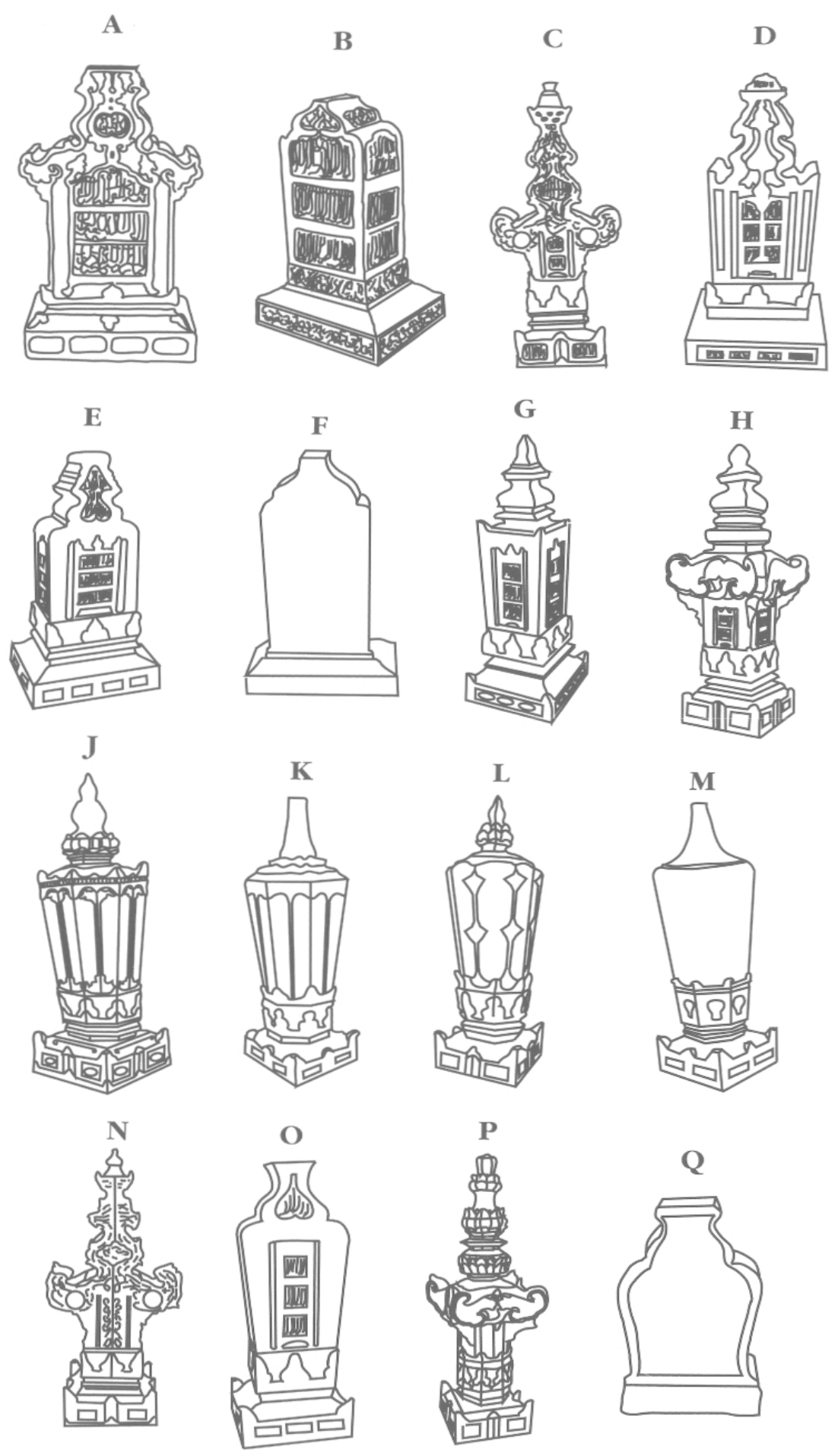

Les types de stèles batu Aceh de l'État de Johor (Malaisie) 


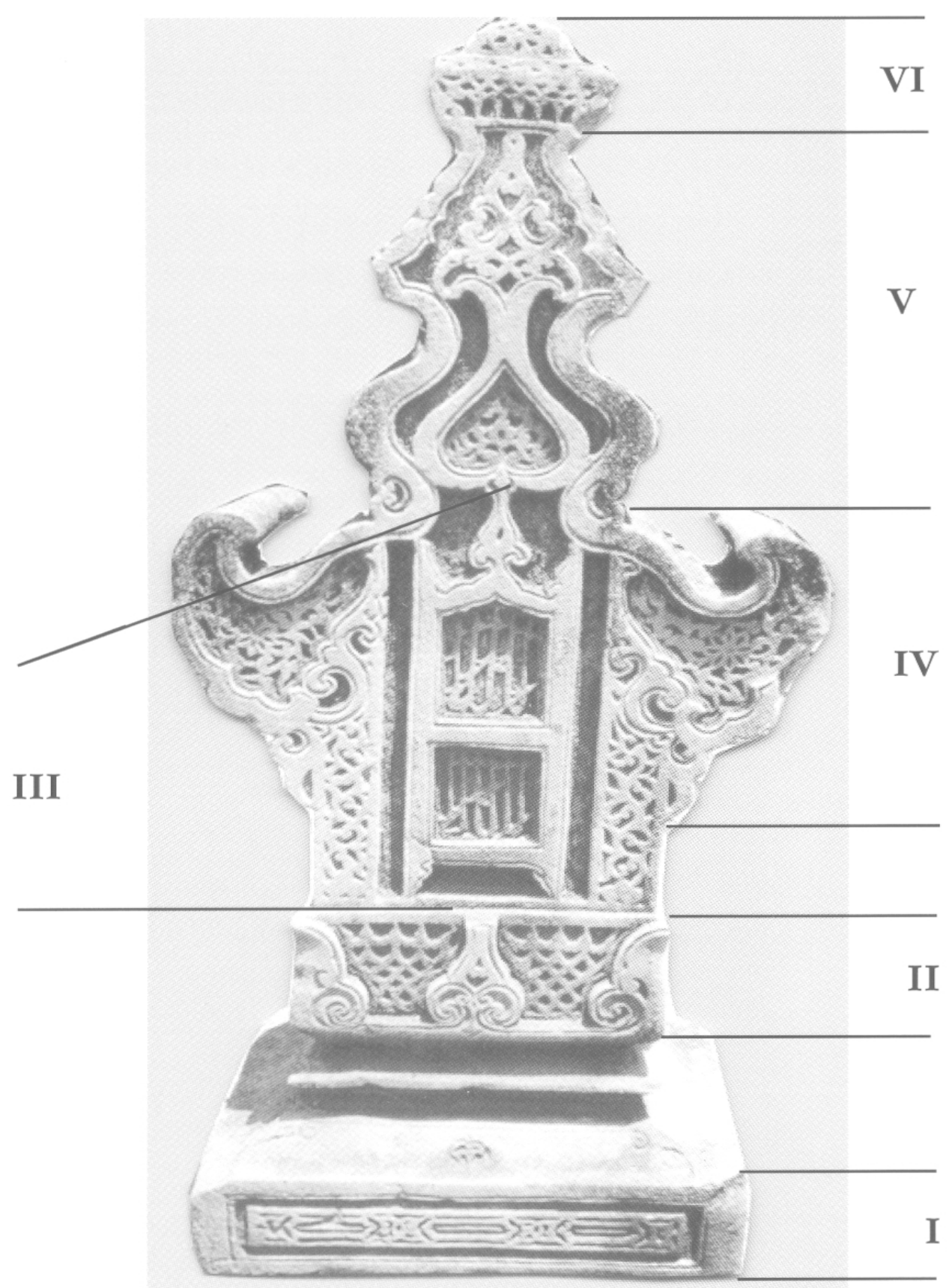

Les différentes parties d'une stèle batu Aceh de type C (hauteur $74 \mathrm{~cm}$, largeur maximale $35 \mathrm{~cm}$ ) 


\begin{tabular}{|c|c|c|c|c|c|c|c|c|c|c|c|c|c|c|c|c|c|c|}
\hline \multirow[b]{2}{*}{ Site } & \multicolumn{17}{|c|}{ Type } & \multirow[b]{2}{*}{ Total } \\
\hline & A & B & C & D & $\mathbf{E}$ & F & G & $\mathrm{H}$ & $\mathbf{J}$ & $\mathbf{K}$ & $L$ & $\mathbf{M}$ & $\mathbf{N}$ & $\mathbf{O}$ & $\mathbf{P}$ & $\mathbf{Q}$ & $?$ & \\
\hline ULU & & & 14 & & & & & 3 & & 1 & & & & 6 & & & & 24 \\
\hline BEN & & & 11 & & & & & & 1 & 7 & & & 1 & & 2 & & & 22 \\
\hline THD & & & 10 & & & & & 2 & & 7 & & 1 & & 1 & & & & 21 \\
\hline SMAH & & & 7 & & 3 & & & 4 & 2 & 2 & & & & & & & 2 & 20 \\
\hline KMAK & & & 10 & 1 & & 1 & & & & 3 & & 1 & & 1 & & & 1 & 18 \\
\hline SPDB & 8 & 1 & 1 & & & 1 & & & & & & & & & & 1 & & 12 \\
\hline SPT & 1 & & & 1 & & 4 & 1 & 1 & & & & & & & & & & 8 \\
\hline SAY & 4 & 1 & & & 1 & 1 & & & & & & & & & & & & 7 \\
\hline KMD & & & 2 & & & & & & & 5 & & & & & & & & 7 \\
\hline BSAW & & & 2 & & & & & 2 & & 2 & 1 & & & & & & & 7 \\
\hline TBEL & & & 2 & 2 & & & & & & & & & & 2 & & & & 6 \\
\hline INAS & 1 & 1 & 3 & & & & & 1 & & & & & & & & & & 6 \\
\hline SELU & & 1 & 2 & & & & & 1 & & & & & & 1 & & & & 5 \\
\hline PAN & & & 2 & & & & & & & & & & 1 & 1 & 1 & & & 5 \\
\hline DRAJ & & & 3 & & & & & & & 2 & & & & & & & & 5 \\
\hline STEL & & & 3 & & & & & & & & & & & 1 & & & & 4 \\
\hline STIR & & & 3 & & & & & & & 1 & & & & & & & & 4 \\
\hline LUK & & & 2 & & & & & & & 2 & & & & & & & & 4 \\
\hline BT & & & 2 & & & & & & & & & & & & & & & 2 \\
\hline JOLA & & & & 1 & & & & & & 1 & & & & & & & & 2 \\
\hline SEPH & & & & 1 & & & & & & 1 & & & & & & & & 2 \\
\hline PRAM & & & 2 & & & & & & & 1 & & & & & & & & 3 \\
\hline LAK & & & 1 & & & & & & & 1 & & & & & & & & 2 \\
\hline KRAJ & 4 & & & & & & & & & & & & & & & & & 4 \\
\hline LUB & & 2 & 1 & & & & & & & & & & & & & & & 3 \\
\hline BCHA & 1 & & & & & & & & & & 1 & & & & & & & 2 \\
\hline SCEK & & & 1 & & & & & & & & & & & & & & & 1 \\
\hline CKD & & & 1 & & & & & & & & & & & & & & & 1 \\
\hline PGD & & & & & & & & & & & & 1 & & & & & & 1 \\
\hline SPEM & & & 1 & & & & & & & & & & & & & & & 1 \\
\hline SIT & & & & & & & & & & & & & 1 & & & & & 1 \\
\hline KBUR & & & & & & & & & & & & & 1 & & & & & 1 \\
\hline KBER & & & 1 & & & & & & & & & & & & & & & 1 \\
\hline BSEN & & & 1 & & & & & & & & & & & & & & & 1 \\
\hline BKER & & & 1 & & & & & & & & & & & & & & & 1 \\
\hline SSLG & & & 1 & & & & & & & & & & & & & & & 1 \\
\hline Total & 19 & 6 & 90 & 6 & 4 & 7 & 1 & 14 & 3 & 36 & 2 & 3 & 4 & 13 & 3 & 1 & 3 & 215 \\
\hline$\%$ & 9 & 3 & 42 & 3 & 2 & 3 & 0 & 7 & 1 & 17 & 1 & 1 & 2 & 7 & 1 & 0 & 1 & 100 \\
\hline
\end{tabular}

Répartition des types de batu Aceh sur les sites de Johor 


\section{Les grands sites à batu Aceh de Kota Tinggi}

Parmi les 211 tombes à batu Aceh recensées à Johor, une seule comporte à la fois l'identité du défunt et la date du décès. Il s'agit de la tombe $\mathrm{n}^{\circ} 3$ du cimetière Sayong Pinang Duabelas (SPDB 3), dont l'épitaphe mentionne « la grand-mère du défunt seigneur Mansûr » ainsi que la date «lundi du mois de sha'bân 857 », soit août-septembre $1453^{29}$. La stèle nord de la tombe $n^{\circ} 1$ de Kampung Raja, Pagoh, (KRAJ 1) comporte quant à elle une épitaphe mentionnant "al-Sultân 'Alâ' al-dîn b. al-Sultân Mansûr Shâh b. al-Sultân Muzaffar Shâh ${ }^{30}$, qui n'est autre que le sultan de Melaka décédé en $1488^{31}$. L'épitaphe de la tombe $\mathrm{n}^{\circ} 10$ du cimetière Sayong Pinang Duabelas (SPDB 10) mentionne un frère de ce sultan, Sulaymân Shâh ${ }^{32}$, qui aurait été assassiné par celui qui deviendra en 1488 Sultan Mahmud Syah de Melaka. À Sayong Pinang, l'épitaphe de la tombe SAY 1 indique le nom de la défunte, To Hang Djumat, et une date incomplète « le mercredi $9^{\mathrm{e}}$ jour qui s'est écoulé du mois de rabî II $"{ }^{33}$. Les autres tombes à batu Aceh de Johor ne comportant ni nom, ni date, les hypothèses concernant leur datation et leur identification reposent uniquement sur des sources écrites, des traditions orales et des analyses comparatives, en partant de l'idée que des tombes qui présentent de nombreuses caractéristiques communes sont contemporaines. Ne disposant pas encore de descriptions précises des batu Aceh pour le reste du monde malais, nos comparaisons avec des stèles extérieures à Johor se font sur la base de documents photographiques. Nous nous intéresserons ici principalement aux grands sites de Kota Tinggi, à savoir celui du bendahara ${ }^{34}$ Tun Habab Abdul Majid (BEN), celui de Sultan Mahmud Mangkat di Julang (SMAH), celui de Makam Tauhid (THD), celui de Ulu Sungai Che Omar (ULU), celui de Kampung Makam (KMAK) et celui de Kampung Makam Dagang (KMD). Ces six sites totalisent 112 tombes, soit $53 \%$ du corpus.

\section{Cimetière du bendahara Tun Habab Abdul Majid (BEN)}

Lors de son expédition au Mont Blumut à la fin des années 1870, Dudley Francis Amelius Hervey s'arrête à Kota Tinggi où il repère deux cimetières ${ }^{35}$ :

«[...] two cemeteries, [...] the other nearly a mile off in the jungle which contains the tombs, some of them handsomely carved, of the bendaharas, the predecessors, it is said, of those who took charge and ultimately became the independent rulers of Pahang ; among these is also the tomb of a YamTuan. It is much to be regretted that none of these tombs have any inscription or even a date. Behind the kampung is Padang Saujana [...].»

La tradition qui fait du cimetière ancien de Padang Saujana le cimetière de la famille du bendahara de Johor est encore bien vivante. Parmi les 22 tombes à batu Aceh de ce complexe, BEN 13 est considérée comme la tombe du bendahara Sri Maharaja Tun Habab Abdul Majid ${ }^{36}$. Les textes malais Tuhfat al-Nafis et Peringatan Sejarah Negeri

29. Ludvik Kalus in Perret et al., 1999 : 127.

30. Ibid. : 110.

31. Linehan, 1973, [ $1^{\text {re }}$ éd., 1936] : 15.

32. Ludvik Kalus in Perret et al., $1999: 142$.

33. Ibid. : 123, 124.

34. Ministre chargé du Trésor, faisant fonction de Premier ministre.

35. Hervey, $1879: 88$.

36. Kamarudin Ab. Razak, $1998:$ 61-62. 
Johor mentionnent qu'il meurt le 7 muharram $1109 \mathrm{H} .{ }^{37}$, soit le 26 juillet 1697 . On sait par ailleurs que la capitale du sultanat est bien située en ce lieu à l'époque, précisément à Makam Tauhid. Ces deux faits s'accordent par conséquent avec la tradition locale. Selon les informations recueillies par Winstedt dans la généalogie des temenggong ${ }^{38}$ de Muar, le bendahara Tun Habab Abdul Majid est l'arrière-petit-fils d'un certain Sayid Idrus, originaire de l'Hadramaout et installé à Aceh ${ }^{39}$. On ne dispose d'aucune information sur l'identité des défunts des 21 tombes à batu Aceh voisines de BEN 13. Il est toutefois probable que s'y trouvent plusieurs de ses 16 enfants ${ }^{40}$.

La comparaison avec des stèles de même type retrouvées ailleurs à Johor fait apparaitre que parmi les 22 tombes de ce cimetière, 15 peuvent être regroupées avec des tombes d'autres sites :

- pour le type C, THD 5 et BEN 14 ont 13 caractéristiques en commun; THD 4 et BEN 17 en ont 9 ; TBEL 3 et BEN 19 en ont 8 ; BEN 1, BEN 6 et BEN 10 ont 7 ou 8 caractéristiques en commun ainsi que 8 avec SCEK 1 ; BEN 8 et KMAK 7 en ont 7 , ainsi que BEN 20 et SMAH 9.

- pour le type K, BEN 3 a 6 caractéristiques en commun avec STIR 1 ; BEN 12 en a 6 avec KMAK 3 et BSAW 1 ; THD 15 a 6 caractéristiques en commun avec BEN 16 et 5 avec BEN 18. PAN 4.

- pour le type N, BEN 11 a 14 caractéristiques en commun avec SIT 1 et 13 avec

- pour le type P, BEN 13 et BEN 21 ont 7 caractéristiques en commun ainsi que 7 avec PAN 5.

Ces comparaisons montrent que plus de la moitié (53\%) des stèles ayant un maximum de caractéristiques communes avec des stèles de BEN se trouvent dans le secteur de Kota Tinggi [THD (3), KMAK (2), SMAH (1), SIT (1)]. Du point de vue statistique, ce résultat est logique, dans la mesure où ce secteur contient près de la moitié $(42 \%)$ de toutes les tombes à batu Aceh recensées à ce jour à Johor.

On ne dispose pas de preuves objectives pour affirmer que BEN 13 est bien la tombe du bendahara Sri Maharaja Tun Habab Abdul Majid. Pour tenter de vérifier cette hypothèse, il faut s'orienter vers la tombe qui, hors de ce cimetière, présente un maximum de similitudes, à savoir PAN 5 sur le site de Panchor, en aval de Kota Tinggi.

Les opinions divergent sur l'identité du défunt de PAN 5 : Sultan Abdul Jalil I ${ }^{41}$, Sultan Abdul Jalil III ${ }^{42}$ ou un bendahara ${ }^{43}$. D'après le Sejarah Melayu, Sultan Abdul Jalil I est enterré à Bukit Seluyut, près de la tombe de Sultan Muzzafar Syah II. À Bukit Seluyut se trouve un ensemble de trois tombes (SELU 1 et SELU 2 [type C], SELU 3 [type B ?]) qui pourraient être en effet liées à des événements relatés dans le Sejarah Melayu. D'après ce texte, le sultan de Johor Muzzafar Syah II meurt à Seluyut et est enterré à Bukit Seluyut, d'où son nom posthume de "Marhum di Seluyud » ${ }^{44}$. Le règne de ce souverain, qui débute probablement en 1564 à la suite d'une attaque acihaise ${ }^{45}$, est très

37. Raja Ali Haji, Tuhfat al-Nafis-Sejarah Melayu-Islam, 1991 : 139 ; Kratz (éd.), 1973 : 44.

38. Haut fonctionnaire chargé de l'ordre urbain à qui le sultan a confié ici un territoire à administrer.

39. Winstedt, 1932a : 58 .

40. Ibid. : 61 .

41. Abdul Halim Nasir, $1990: 111,112$ (pl. 7.2) et 113 (pl. 7.3).

42. Généalogie royale de Johor/Pahang/Riau détenue par Raja Kamarazaman bin Raja Salim, Kota Tinggi.

43. Abdullah bin Abu Talib (Panchor), communication personnelle du 19/12/1995.

44. Abdul Samad bin Ahmad (éd.), $1986: 307$; Shellabear (éd.), $1994: 235$.

45. Mendes Pinto, 1991, [ [1 éd. 1614]: 113. 
peu documenté. On sait seulement qu'il se rend à Melaka en mars $1568^{46}$. Deux sources portugaises datées d'août et de septembre 1569 indiquent d'une part qu'il réside un certain temps à Johor Lama après 1564, d'autre part qu'un autre sultan, Sultan Abdul Jalil Syah II, est à présent sur le trône ${ }^{47}$. Muzzafar Syah II meurt donc probablement avant août 1569. Selon le Sejarah Melayu, c'est son neveu, Sultan Abdul Jalil I, qui lui succède, mais celui-ci meurt à l'âge de neuf ans, peu de temps après avoir accédé au trône. Il est également enterré à Bukit Seluyut, près de la tombe de Sultan Muzzafar Syah II ${ }^{48}$. Comme pour Sultan Muzzafar Syah II, la date précise du décès de Sultan Abdul Jalil I n'est pas connue et l'on peut seulement conclure qu'il meurt avant août 1569, puisque son successeur est déjà sur le trône. Toujours selon le Sejarah Melayu, Raja Fatimah, fille de Sultan Alauddin Riayat Syah II et sœur cadette de Sultan Muzzafar Syah II, est également enterrée à Bukit Seluyut, près de la tombe de son frère ${ }^{49}$. Cet événement se situe avant la mort de Sultan Abdul Jalil Syah II en 1597.

En suivant le texte malais, qui mentionne donc l'existence de trois tombes regroupées à Bukit Seluyut, on est tenté de penser que les tombes SELU 1, SELU 2 et SELU 3 abritent les trois personnes mentionnées ci-dessus, à condition bien sûr que les stèles soient encore à leur place d'origine. Bien que SELU 2 soit endommagée, nous avons pu recenser quatorze détails ornementaux communs avec SELU 1 et l'on peut dire qu'à l'exception des dimensions qui diffèrent quelque peu, les stèles sont identiques. Ces similitudes s'accordent bien avec l'hypothèse de tombes de germains, c'est-à-dire Sultan Muzzafar Syah II et Raja Fatimah. Par ailleurs, bien qu'elle soit très endommagée, la stèle qui subsiste pour SELU 3 est clairement une petite stèle qui conviendrait parfaitement à une tombe d'enfant, en l'occurrence Sultan Abdul Jalil I. Si l'on compare les stèles de ces trois tombes avec des photos de stèles en dehors de Johor, SELU 1 et SELU 2 ressemblent à une stèle de la tombe de Tok Subang située à Kampung Kandang ${ }^{50}$ près de Sayong, Kuala Kangsar, Perak. La tradition fait de Tok Subang une concubine de Sultan Mansur Syah I de Perak (1549 ?-1577 ?), qui la fera assassiner. Bien qu'incertaine, la datation proposée pour le règne de ce sultan s'accorde avec la datation proposée pour les trois tombes de Seluyut.

Toutes ces informations nous amènent à penser que PAN 5 n'est pas la tombe de Sultan Abdul Jalil I. Sultan Abdul Jalil Syah III meurt, quant à lui, à Pahang ${ }^{51}$. La tradition qui veut que PAN 5 soit la tombe d'un bendahara nous paraît par contre beaucoup plus solide. Une source locale, le Peringatan Sejarah Negeri Johor, mentionne en effet que le bendahara Tun Mas Anom meurt à l'époque où la capitale est à Panchor ${ }^{52}$. Ce bendahara est un frère cadet de Sultan Abdul Jalil IV ${ }^{53}$ et une source hollandaise indique qu'il meurt le 29 mars $1708^{54}$. La similitude des tombes PAN 5 et BEN 13 pourrait alors s'expliquer par le fait que Tun Habab Abdul Majid n'est autre que le père du bendahara Tun Mas Anom.

Une autre hypothèse de datation indirecte nous paraît envisageable à partir des similitudes observées entre BEN 11 et PAN 4, à nouveau donc sur le site de Panchor.

46. Diogo do Couto, cité par Gibson-Hill, $1955: 146$.

47. Sousa Pinto, 1994 : 146.

48. Abdul Samad bin Ahmad (éd.), $1986: 307-308$; Shellabear (éd.), $1994: 235-236$.

49. Abdul Samad bin Ahmad (éd.), $1986: 310$. Shellabear (éd.), $1994: 238$.

50. Abdul Halim Nasir, 1977 : 125 ; Othman bin Mohd. Yatim, 1988 : 171 (pl. 11), 214 (pl. 54).

51. Andaya, $1975: 114$.

52. Kratz (éd.), $1973: 46$.

53. Andaya, $1975: 201$.

54. Ibid. : 211. 
Remontant la rivière Johor en 1826 , un groupe venu de Singapour s'arrête près de Bukit Sungai Tukul (Panchor) et note ${ }^{55}$ :

«[...] At the N.E. end of the fort we found a tomb formed by piling up large flat stones and filling up the centre with earth; there were two stones standing erect about a foot apart ; they were of the same form, 3 feet high, very handsomely carved, and in good state of preservation. They are of hard sandstone and said by our guide (Inchi Salle) to be the workmanship of a Chinese and to be the tomb of Raja Tungko Putih; but he could not tell us when he lived or anything of his history.»

Cette tombe n'est autre que PAN 4 (type N), dont le nom subsiste aujourd'hui avec quelques variantes : Raja Puteh ${ }^{56}$, Puteri Putih ${ }^{57}$, Tengku Dara Putih ou encore Tengku Putih $^{58}$. Tous ces noms renseignent peu sur le défunt, si ce n'est qu'il s'agit probablement d'une femme dont le titre "Tengku » indiquerait qu'elle fait partie de la famille d'un sultan. On sait que le sultan Abdul Jalil IV s'installe à Panchor après le mois d'octobre $1701^{59}$ et avant le mois de décembre $1705^{60}$. D'après le témoignage de Walter Vaughan, un agent de l'East India Company, il semble que Panchor soit déjà la capitale en $1703^{61}$. La défunte serait donc à rechercher dans la famille de ce sultan. Le même Vaughan rapporte un événement qui pourrait bien avoir un rapport direct avec la tombe PAN 4. Il mentionne en effet que la sœur aînée du sultan Abdul Jalil IV, épouse du syahbandar ${ }^{62}$, meurt en couches le 4 septembre $1703^{63}$. Il est très probable que ce soit le personnage appelé Tengku Putih dans les traditions locales. Une généalogie conservée par un descendant de Sultan Abdul Jalil IV conforte cette hypothèse en mentionnant que Raja (ou Tengku) Dara Puteh, fille de Sultan Abdul Jalil Syah III, meurt à Panchor et prend le nom posthume de « Marhumah Mangkat di Bukit Panchor».

La stèle PAN 4 a de nombreuses similitudes avec SIT 1, également de type N, située à Kampung Tembeyoh près de Kota Tinggi. La tradition locale veut que cette tombe abrite une femme ayant vécu à l'époque de Sultan Mahmud Syah II (fin du XVII ${ }^{\mathrm{e}}$ siècle). Les datations proposées pour PAN 4 et SIT 1 conviendraient donc bien également pour BEN 11, en particulier si l'on admet que le cimetière du bendahara Tun Habab Abdul Majid a été ouvert après l'installation de Sultan Mahmud Syah II à Makam Tauhid en juillet 1688 .

En Malaisie, la plupart des batu Aceh de type $\mathrm{N}$ se trouvent dans le cimetière royal de Kampung Langgar à Kedah (9 tombes) ${ }^{64}$. Ce cimetière est ouvert en 1701 sur ordre de Sultan Abdullah Al-Muazzam Shah, une date qui correspond tout à fait aux datations proposées ci-dessus. Il y a néanmoins une incertitude sur la datation des tombes de ce cimetière, car des stèles plus anciennes y ont été transportées ${ }^{65}$, y compris peut-être les stèles de type $\mathrm{N}$. Nous disposons par ailleurs d'une date précise de stèle de type $\mathrm{N}$ hors de

55. Viator in Moor, $1837: 265$.

56. Winstedt, $1992: 213$.

57. Abdul Halim Nasir, $1990: 111$.

58. Norjihan Nordin, $1994: 38$.

59. Deux émissaires hollandais arrivent le 6 octobre 1701 à Panchor où ils rencontrent d'abord le sultan Abdul Jalil IV avant de remonter la rivière pour atteindre la capitale (Andaya, 1975 : 199, 242 note 9). Cependant, selon le texte Hikayat Negeri Johor, ce sultan s'installe à Panchor une année seulement après le début de son règne, soit fin 1700 (Ismail Hussein éd., $1992: 236$ ).

60. Andaya, $1975: 205$.

61. Rapport de Walter Vaughan in Winstedt, $1992: 216-220$.

62. Haut fonctionnaire chargé des douanes et de la surveillance des marchands étrangers.

63. Cité par Winstedt, $1992: 219$.

64. Othman bin Mohd. Yatim, $1988: 43$.

65. Ibid. : 118 . 
Malaisie : il s'agit de la tombe du sultan Ageng Tirtayasa de Banten ${ }^{66}$, qui meurt en 1692, là encore une date qui s'intercale dans la fourchette de datation des trois tombes de Johor.

\section{Cimetière de Sultan Mahmud Mangkat di Julang (SMAH)}

Après s'être arrêté à Panchor, le même groupe de visiteurs de Singapour remonte la rivière Johor et fait halte à Kota Tinggi, où un cimetière retient leur attention ${ }^{67}$ :

«The platform which forms the burial-place is 30 yards long by 10 broad; raised about 5 feet, and built round with brick and mortar. [...] There are sixteen pairs of tombstones; one of these is longer than the rest and they are all of the same shape nearly; but from their weathered appearance, they must be of more ancient date [...] The larger stones form the tomb of Sultan Mahomet the last king that was buried here. [...] Our guide informed us that there are remains of a ditch which runs a considerable way into the jungle and includes the burial ground : the place is called Kota tingeh.»»

Un demi-siècle plus tard, Hervey visite le même endroit et observe ${ }^{68}$ :

«[...] two cemeteries, one close to the kampung surrounded by a low wall of laterite and containing the tombs of the formers sovereigns $[\ldots]$ ».

Selon une tradition recueillie par Winstedt ${ }^{69}$, c'est le mufti de Johor qui, à l'occasion d'un rêve, a connaissance de l'identité des souverains enterrés au cimetière de Makam Sultan Mahmud Mangkat di Julang (SMAH), à savoir Sultan Alauddin, Sultan Abdullah, Sultan Abdul Jalil et Sultan Mahmud. Cette vieille tradition perdure, puisque aujourd'hui encore le cimetière est appelé Makam Sultan Mahmud Mangkat di Julang. Sur le site, un plan avec des explications en $j a w i{ }^{70}$ donne les identifications suivantes: SMAH 1 est la tombe de Sultan Abdullah Syah ibn Sultan Muzzafar Syah, SMAH 2 la tombe de Sultan Alauddin Riayat Syah, SMAH 5 la tombe de Sultan Abdul Jalil Syah V, SMAH 6 la tombe de Satilah Salam (descendance de Sultan Mahmud), SMAH 7 la tombe de Sultan Mahmud Syah ibn Sultan Ibrahim Syah. SMAH 3, SMAH 4, SMAH 11 et SMAH 14 seraient les tombes d'hommes non identifiés alors que SMAH 8, SMAH 9, SMAH 10, SMAH 12, SMAH 13 et SMAH 15 seraient les tombes de femmes non identifiées ${ }^{71}$.

Les sources malaises mentionnent effectivement que Sultan Mahmud Syah II meurt à Kota Tinggi en $1111 \mathrm{H}$. (1699-1700 E.C.) ${ }^{72}$, ce que confirme une source hollandaise d'octobre $1699^{73}$. Ainsi, comme pour BEN 13, bien qu'on manque de preuves objectives pour affirmer que SMAH 7 est effectivement la tombe de Sultan Mahmud Syah II, la tradition locale ne peut être rejetée d'emblée.

Les stèles de SMAH 7 sont de type $\mathrm{H}$, un type que l'on trouve à Aceh au moins dès 1530 avec notamment la tombe du sultan Ali Mughayat Syah qui meurt le 7 août $1530^{74}$. En dehors de l'État de Johor, le site de Malaisie le plus riche en stèles de ce type est Makam Chondong à Pahang (4 tombes) ${ }^{75}$. Elles constituent une partie de la cargaison de stèles envoyées d'Aceh sur ordre du sultan Iskandar Thani en 1638-1639, comme il est

66. Ambary, $1984: 416,417$ (photo 13), 418 (photo 15).

67. Viator in Moor, $1837: 268$.

68. Hervey, $1879: 88$.

69. Winstedt, $1992: 210$.

70. Écriture arabe adaptée à la notation du malais.

71. Kamarudin Ab. Razak, 1998 : 56-57.

72. Raja Ali Haji, Tuhfat al-Nafis-Sejarah Melayu-Islam, 1991 : 140 ; Kratz (éd.), 1973 : 44 ; Mohd. Yusof Md. Nor (ćd.), $1984: 31$.

73. Andaya, $1975: 186$.

74. Ambary, $1984: 418$ et photo 24 ; Othman bin Mohd. Yatim, $1988: 32,167$ (photo a) ; Lombard, $1967: 185$.

75. Othman bin Mohd. Yatim, $1988: 43$. 
noté dans le Bustan al-Salatin ${ }^{76}$. Par ailleurs, d'après Othman bin Mohd. Yatim, les stèles de types $\mathrm{H}$ sont encore utilisées à Aceh dans les années $1760^{77}$. Leur usage s'étendrait ainsi sur plus de deux siècles.

Comparée à toutes les stèles de type $\mathrm{H}$ recensées à Johor, SMAH 7 a le plus de caractéristiques communes (13) avec SMAH 5, mais le décor de SMAH 5 est plus soigné. La tradition locale veut que SMAH 5 soit la tombe du sultan Abdul Jalil Syah V. Mais selon le texte Tuhfat al-Nafis et une source étrangère, ce souverain meurt en janvier 1761 et est enterré à Batangan (Riau) près de la tombe de son père ${ }^{78}$, ce qui rend la tradition locale difficile à accepter.

Une autre possibilité doit être envisagée, à savoir que le site de Makam Sultan Mahmud Mangkat di Julang abriterait la tombe du sultan Ala Jalla Abdul Jalil Syah II (Raja Omar). Le Sejarah Melayu mentionne ainsi qu'après s'être déplacé vers l'amont de la rivière Damar, ce sultan fait édifier une enceinte en briques qu'il appelle Makam Tauhid, à l'intérieur de laquelle il sera enterré, d'où son nom posthume de «Marhum Mangkat di Batu $"{ }^{79}$. Au cours des années 1940, H. G. Q. Wales a dégagé ce qu'il a cru être les fondations d'un bâtiment en briques entre le site de Makam Sultan et la rivière Johor ; il observe à cette occasion que les tombes du site de Makam Sultan sont entourées de briques ${ }^{80}$. Cette abondance de briques n'est pas sans rappeler la mention du Sejarah Melayu, d'autant plus que selon une tradition locale, Makam Tauhid était situé à proximité de l'endroit appelé aujourd'hui Kampung Makam Dagang, par conséquent non loin du cimetière Sultan Mahmud Mangkat di Julang. L'emplacement originel de la tombe de Sultan Ala Jalla Abdul Jalil Syah II a pu ainsi être proche de ce cimetière et sa tombe y être transférée à un moment donné. Sultan Ala Jalla Abdul Jalil Syah II meurt en 1597, soit 102 ans avant Sultan Mahmud Syah II. Si cet intervalle n'est pas un obstacle en luimême pour fabriquer SMAH 7 suivant le modèle de SMAH 5, on comprend moins cependant pourquoi cette nouvelle stèle serait moins raffinée que la première. La seule conclusion provisoire que l'on peut tirer de ces comparaisons est que dans le cimetière Sultan Mahmud Mangkat di Julang, la tombe la plus raffinée est SMAH 5, et ce fait doit être pris en considération avant d'émettre un quelconque avis sur l'identité des personnes enterrées à cet endroit.

Une tradition locale veut que SMAH 1 soit la tombe de Sultan Abdullah Syah ibn Sultan Muzzafar Syah, mais elle est en contradiction avec trois sources différentes indiquant que ce souverain est mort dans l'archipel de Tambelan (Riau) ${ }^{81}$. De même, la tradition locale identifie SMAH 2 comme la tombe de Sultan Alauddin Riayat Syah. Nous avons vu plus haut que la tombe de Sultan Alauddin Riayat Syah I se trouve à Kampung Raja, Pagoh (KRAJ 1). Deux sources d'origines distinctes mentionnent par ailleurs que Sultan Alauddin Riayat Syah II est fait prisonnier ou meurt à Aceh ${ }^{82}$. Si la date et le lieu de décès de Sultan Alauddin Riayat Syah III ne sont pas connus avec certitude, plusieurs

76. Linehan, 1973 [ $1^{\text {re }}$ éd. 1936] : 37-38.

77. Othman bin Mohd. Yatim, $1988: 37$.

78. Raja Ali Haji, The Precious Gift. Tuhfat al-Nafis, 1982 : 116-117.

79. Abdul Samad bin Ahmad (éd.), $1986: 310$; Shellabear (éd.), 1994 : 237-238.

80. Wales, $1940: 62-63$. Selon une hypothèse récente, il pourrait s'agir des fondations d'une mosquée du XVII ${ }^{\mathrm{e}}$ s. (Abdul Halim Nasir, $1990: 61$ ).

81. Raja Ali Haji, Tuhfat al-Nafis-Sejarah Melayu-Islam, 1991 : 139 ; Maxwell, 1882 : 98 ; lettre de Cornelis Simonsz van der Veer au Gouverneur général Antonie van Diemen du 12/12/1637 (annexe I dans Leupe, $1936: 149$ ).

82. Maxwell, 1882 : 97-98; Mendes Pinto, $1991: 113$. 
sources permettent de penser qu'il n'est pas enterré à Kota Tinggi ${ }^{83}$. Il est par conséquent très peu probable que SMAH 2 soit la tombe d'un sultan de Melaka ou de Johor portant le nom de Sultan Alauddin Riayat Syah.

Parmi les vingt tombes à batu Aceh du cimetière Sultan Mahmud Mangkat di Julang, six présentent des similitudes significatives avec des tombes de même type à Johor :

- pour le type C, SMAH 13 présentent de nombreux traits communs avec THD 1 (11), ULU 24 (10) et KMAK 14 (10); SMAH 15 avec SMAH 8 (10) et ULU 20 (10) ; SMAH 9 avec BEN 20 (7) ainsi qu'avec THD 14 (7) ;

- pour le type H, SMAH 18 présente de nombreux traits communs avec INAS 3 (4);

- pour le type K, SMAH 3 présente de nombreux traits communs avec BEN 3 (5), STIR 1 (5), BEN 12 (5), KMAK 3 (5) et BSAW 1 (5).

Comparé au cimetière voisin de $\mathrm{BEN}$ où plus des deux tiers des tombes peuvent être valablement rapprochées de tombes d'autres cimetières, SMAH présente donc une situation assez particulière avec ce faible nombre. Si l'on considère le nombre de tombes similaires, c'est BEN qui semble le plus proche de SMAH, avec trois tombes comparables. Néanmoins, si l'on prend en compte le nombre de traits communs avec ces trois tombes, le lien entre SMAH et BEN n'est pas très étroit. BEN 20 possède 7 traits communs avec SMAH 9 ; BEN 3 et BEN 12 en ont quant à eux seulement 5 avec SMAH 3. Le lien est plus étroit avec deux tombes du cimetière de Ulu Sungai Che Omar : ULU 24 (10 traits communs avec SMAH 13) et ULU 20 (10 traits communs avec SMAH 15).

SMAH possède par ailleurs trois des quatre tombes à stèles de type $E$ de Johor, la quatrième se trouvant à Sayong Pinang. Hors de Johor, on retrouve ce type dans le cimetière Sultan Muzaffar Syah I à Perak. Si l'on retient l'époque du décès de ce sultan, soit le milieu du XVI ${ }^{\mathrm{e}}$ siècle ${ }^{84}$, comme indication pour dater les trois tombes du même type de SMAH, on en déduit que SMAH a fonctionné comme cimetière de hauts personnages du sultanat de Johor bien avant le règne de Sultan Mahmud Syah II à Kota Tinggi (16881699), sultan dont le nom est associé à ce cimetière. Il est par conséquent possible que ce cimetière ait fonctionné au moins dès la première période où le secteur de Kota Tinggi a abrité la capitale du sultanat de Johor, c'est-à-dire entre 1589 et 1617.

\section{Makam Tauhid (THD)}

L'endroit aujourd'hui appelé Makam Tauhid était autrefois généralement désigné sous le nom de Makam Sayid, comme c'est le cas dans la description faite par Winstedt dans les années $1930^{85}$, puis dans les travaux plus récents de Othman bin Mohd. Yatim et de Shaharom Husain ${ }^{86}$. À notre connaissance, l'origine du nom Makam Sayid n'a jamais été expliquée. Il pourrait s'agir d'individus portant le nom de Sayid en raison de leur ascendance, peut-être des marchands ou des ulémas installés à Johor. Considérant l'information obtenue par Winstedt du temenggong de Muar selon laquelle le bendahara Tun Habab Abdul Majid est un arrière-petit-fils de Sayid Idrus originaire de l'Hadramaout, il est plausible qu'une communauté de Sayid soit installée à Kota Tinggi à la fin du XVII ${ }^{\mathrm{e}}$ siècle.

83. Maxwell, 1882 : 98 ; Foster (éd.), 1995 [1 1 éd. 1934] : 58-59, 169 ; Marsden, 1986 [1 $1^{\text {re }}$ éd. 1783] : 440 ; Netscher, 1870:30 ; Tiele, 1886: 303 ; Terpstra, 1938: 71 ; Gibson-Hill, 1956: 128 ; Sousa Pinto, $1994: 148$.

84. Othman bin Mohd. Yatim, $1988: 41$.

85. Winstedt, $1992: 210-211$.

86. Othman bin Mohd. Yatim, $1988: 43$, pl. 42 et 43 ; Shaharom Husain, $1995: 179$. 
Parmi les vingt et une tombes à batu Aceh de Makam Tauhid, douze présentent des similitudes significatives avec des tombes à batu Aceh de même type à Johor :

- pour le type C, THD 5 et BEN 14 présentent de nombreux traits communs (13); THD 4 et BEN 17 présentent de nombreux traits communs (9); THD 13 présente de nombreux traits communs avec BT 2 (10) et SPDB 1 (8); THD 1 présente de nombreux traits communs avec SMAH 13 (11) et ULU 24 (10); THD 12 présente de nombreux traits communs avec ULU 20 (10); THD 18 présente de nombreux traits communs avec KMAK 2 (9); THD 21 présente de nombreux traits communs avec KMAK 6 (8); THD 11 présente de nombreux traits communs avec PAN 3 (8); THD 14 présente de nombreux traits communs avec SMAH 9 (7);

- pour le type K, THD 15 présente de nombreux traits communs avec BEN 16 (6) et BEN 18 (5) ; THD 7 présente de nombreux communs avec KMAK 16 (5);

- pour le type O, THD 10 présente de nombreux traits communs avec ULU 18 (4).

La plupart des stèles ayant un maximum de caractéristiques communes avec des stèles de THD se trouvent dans le secteur de Kota Tinggi [BEN (4), KMAK (3), ULU (3), SMAH (2)]. Du point de vue statistique, ce résultat est logique, dans la mesure où ce secteur contient près de la moitié $(42 \%)$ de toutes les tombes à batu Aceh recensées à ce jour à Johor.

\section{Ulu Sungai Che Omar (ULU)}

Dans l'état actuel des connaissances, ce cimetière est le plus grand complexe funéraire à batu Aceh de Malaisie. Il est curieux cependant qu'il n'existe apparemment ni traditions orales, ni données dans les sources écrites concernant ce site, à propos duquel Winstedt a émis deux hypothèses. Selon sa première hypothèse, l'endroit s'appelait autrefois Pasir Raja ou Pasar Raja et aurait abrité la capitale du sultanat pendant un certain temps entre 1597 et 1613. Selon sa seconde hypothèse, la capitale est à nouveau là lorsque le sultan de Johor change le nom de celle-ci (Makam Tauhid devenant Batu Sawar) et la transfère de l'autre côté de la rivière. Si cette dernière hypothèse est vraie, le site aurait abrité la capitale du sultanat de Johor entre 1642 et avril 1673, date de sa destruction par Jambi.

Néanmoins, deux éléments permettent de douter du bien-fondé de ces deux hypothèses. Il n'existe aucun toponyme Pasir Raja, Pasar Raja ou Batu Sawar à proximité du cimetière Ulu Sungai Che Omar, que ce soit dans le compte rendu de l'expédition d'Hervey dans les années 1870 ou à l'heure actuelle, alors que de nombreux toponymes anciens sont encore en usage dans la région de Kota Tinggi. Par ailleurs, nous n'avons observé aucun témoignage d'habitat ancien à proximité du site, en particulier sous forme de tessons de céramiques importées.

Nous sommes plus enclins à penser que ce n'est pas le centre de la capitale qui se trouvait à proximité du site, mais plutôt un quartier ou village administré par un haut personnage du sultanat ou encore un village ou quartier de hauts personnages " étrangers ». Cette hypothèse est conforme à la morphologie des capitales malaises anciennes, qui sont constituées de plusieurs "villages» (kampung) aux environs du complexe palatin. L'île appelée Pulau Pahang sur la rivière Johor à moins de deux kilomètres en aval du site, ainsi qu'un débarcadère à quelques centaines de mètres en aval sur la même rive, mentionnés par Hervey ${ }^{87}$, marquent peut-être la présence à une époque ancienne de populations étrangères au sultanat. L'absence d'une quelconque information orale ou écrite, locale ou étrangère, sur l'histoire de ce site important est troublante ; elle 
est peut-être liée à un scandale ou à une tragédie que les sources locales ont sciemment omis et que les étrangers auraient ignorés.

Parmi les vingt-quatre tombes à batu Aceh de Ulu Sungai Che Omar, dix présentent des similitudes significatives avec des tombes à batu Aceh de même type à Johor :

- pour le type C: ULU 23 présente de nombreux traits communs avec DRAJ 1 (13) et LAK 1 (13); ULU 22 présente de nombreux traits communs avec LAK 1 (10) et BEN 17 (10); ULU 24 présente de nombreux traits communs avec THD 1 (10) et SMAH 13 (10); ULU 20 présente de nombreux traits communs avec KMAK 14 (10), THD 12 (10), SMAH 15 (10) et KMD 1 (10); ULU 1 présente de nombreux traits communs avec KMD 1 (10); ULU 4 présente de nombreux traits communs avec TBEL 6 (10);

- pour le type O : ULU 14 est le plus proche de TBEL 5 (6) et ULU 21 (5); ULU 10 le plus proche de KMAK 18 (4) et STEL 3 (4); ULU 18 le plus proche de THD 10 (4).

Comme pour THD, SMAH et dans une moindre mesure BEN, la plupart des stèles ayant un maximum de caractéristiques communes avec des stèles de ULU se trouvent dans le secteur de Kota Tinggi [LAK (2), BEN (1), THD (3), SMAH (2), KMAK (2), KMD (2)]. Sans information permettant de dater directement le site, seules les comparaisons de stèles peuvent fournir des éléments de datation. Nous avons signalé ci-dessus les similitudes entre ULU 22, ULU 23 et LAK 1. Selon les traditions locales, LAK 1 est la tombe de Megat Seri Rama, l'assassin de Sultan Mahmud Syah II en 1699, ou de son épouse qui est assassinée peu avant par ce même sultan. ULU 2 et LAK 1 présentent par ailleurs de nombreuses similitudes avec BEN 17. Si l'on admet que BEN est ouvert en 1688 lorsque Sultan Mahmud Syah II s'installe à Makam Tauhid, ULU 22 et ULU 23 pourraient bien dater de la fin du XVII $\mathrm{e}$.

Une autre donnée intéressante concernant ce site est le nombre de tombes à stèles de type $O$ ( 6 unités), le plus élevé en ce qui concerne Johor. On retrouve ce type de stèle dans six autres sites de l'État: THD, KMAK, TBEL, SELU, PAN, STEL. Si STEL 3 est contemporain de l'installation de la capitale du sultanat à Pekan Tua (ca. 1533-1535), alors une ou plusieurs des stèles de type $\mathrm{O}$ de ULU pourraient être datées de la première moitié du XVI ${ }^{\mathrm{e}}$ siècle. Si l'on considère par ailleurs que SELU 5, de type $\mathrm{O}$, date de la même époque que les tombes SELU 1, SELU 2, SELU 3 et SELU 4, alors une ou plusieurs des stèles de type $\mathrm{O}$ de ULU pourraient être datées de la seconde moitié du XVI ${ }^{\mathrm{e}}$ siècle.

\section{Kampung Makam (KMAK)/Kampung Makam Dagang (KMD)}

Parmi les vingt-cinq tombes à batu Aceh de ces deux ensembles, douze présentent des similitudes significatives avec des tombes à batu Aceh de même type à Johor :

- pour le type C: KMAK 14 présente de nombreux traits communs avec SMAH 13 (10) et ULU 20 (10); KMD 1 présente de nombreux traits communs avec ULU 20 (10) et ULU 1 (10); KMAK 2 présente de nombreux traits communs avec THD 18 (9); KMAK 6 et KMAK 15 présentent de nombreux traits communs avec THD 21 (8); KMAK 7 présente de nombreux traits communs avec BEN 8 (7); KMAK 17 présente de nombreux traits communs avec LUK 2 (7);

- pour le type D : KMAK 9 est le plus proche de JOLA 1 (8) et TBEL 1 (7);

- pour le type F : KMAK 10 est le plus proche de SAY 5 (5);

- pour le type K: KMAK 3 est le plus proche de BEN 12 (6) et BSAW 1 (6); KMAK 16 est le plus proche de THD 7 ;

- pour le type O : KMAK 18 est le plus proche de STEL 3 (5). 
Nous avons montré ailleurs que le secteur de Kota Tinggi a abrité la capitale du sultanat de Johor à trois reprises : 1589-1617, 1641-1673 et 1688-1702 ${ }^{88}$. Apparemment, d'après les comparaisons entre stèles, il semble difficile d'attribuer un site de ce secteur à une seule des trois périodes. Des cimetières comme ULU, BEN, THD et SMAH sembleraient donc avoir été utilisés continuellement ou presque à partir de la première installation de la capitale. Si c'est le cas, les noms des cimetières Bendahara Tun Habab Abdul Majid et Sultan Mahmud Mangkat di Julang prêtent à confusion sur le plan chronologique, car ces sites auraient en fait été utilisés bien avant le décès de ces deux personnages. Il faut reconnaître également que la chronologie des styles de batu Aceh n'est pas encore suffisamment précisée pour qu'on puisse déterminer avec certitude si un site correspond à l'une des trois périodes ou non.

Par ailleurs, si l'on compare les tombes de ULU, THD et SMAH avec celles de BEN, on constate que seul THD possède plus d'une tombe ayant de nombreuses similitudes significatives avec une tombe de BEN. Bien que ULU soit le plus grand cimetière à batu Aceh de Johor, aucune tombe de ce site n'a de ressemblance significative avec une tombe de BEN. Les similitudes entre BEN et THD signifient-elles que ces deux sites sont complètement ou partiellement contemporains et à l'inverse l'absence de similitudes entre BEN et ULU signifie-t-elle que les deux sites sont utilisés à des périodes différentes ? Une réponse certaine est très difficile. BEN et SMAH par exemple, qui, si l'on s'en réfère au nom des sites, devraient être absolument contemporains, ne présentent de similitudes significatives que pour une tombe de chaque site. Si l'on considère d'une part ces divergences, d'autre part le fait que les personnages principaux des deux sites seraient respectivement un sultan et un bendahara, l'absence ou le petit nombre de tombes similaires entre deux sites pourraient s'expliquer par autre chose que la chronologie, à savoir par la différence de position sociale des défunts.

\section{Conclusion}

Les diverses prospections effectuées en Malaisie dans l'État de Johor entre 1996 et 1999 ont permis de recenser 36 sites de tombes musulmanes à stèles dites batu Aceh, soit quinze sites supplémentaires par rapport à une recherche similaire effectuée durant les années 1980 , le nombre de tombes recensées passant à 211 unités, soit une augmentation de plus de $50 \%$. Dans l'état actuel des connaissances, l'État de Johor est ainsi de loin la région de la péninsule Malaise la plus riche en tombes de ce genre. Ces prospections ont également permis de confirmer que parmi les genres de tombes musulmanes les plus anciennes recensées à Johor, les tombes à batu Aceh sont de très loin les plus nombreuses. Cette constatation peut très probablement être étendue à toute la péninsule Malaise et à Sumatra. Si l'on ajoute à cela une variété de types et une richesse de décors sans équivalent ainsi que, sur un plan général, la rareté des vestiges dans la région, les batu Aceh constituent un matériau exceptionnel pour l'histoire de l'islam en général et de l'art islamique en particulier dans le monde malais.

Des descriptions, les plus précises et les plus systématiques jamais effectuées sur ce genre de stèles, permettent de compléter la typologie existante basée sur la forme générale, d'enregistrer les nombreuses variantes de décors pour chaque type et de repérer les éléments décoratifs communs à deux ou plusieurs types. Ces descriptions facilitent par ailleurs des comparaisons entre toutes les stèles d'un même type; ces comparaisons permettent à leur tour de regrouper les stèles présentant un nombre significatif de

88. Perret et al., $1999:$ 159-169, 173. 
caractéristiques similaires pour aider à l'interprétation chronologique et historique dans un contexte de très grande rareté d'inscriptions à caractère historique. Ainsi, des hypothèses sur la chronologie des types et l'identification de tombes peuvent être proposées, comme nous avons tenté de le faire ici à propos des grands sites de Kota Tinggi.

Le corpus des batu Aceh de Johor montre enfin que les problèmes de chronologie et de diffusion des différents types de batu Aceh ainsi que leur choix en rapport avec la position sociale du défunt ne pourront être éclaircis sans un enrichissement du corpus à des fins comparatives en effectuant, pour toutes les autres batu Aceh du reste du monde malais, une description systématique similaire à celle qui vient d'être réalisée à Johor.

\section{Références citées}

Abréviations

$\begin{array}{ll}\text { BKI } & \text { Bijdragen tot de Taal-, Land-en Volkenkunde } \\ \text { JMBRAS } & \text { Journal of the (Malayan) Malaysian Branch of the Royal Asiatic Society } \\ \text { JSBRAS } & \text { Journal of the Straits Branch of the Royal Asiatic Society } \\ \text { VBG } & \text { Verhandelingen van het Bataviaasch Genootschap van Kunsten en Wetenschappen }\end{array}$

ABDUL HALIM NASIR

1977 Sejarah Perak, Kuala Lumpur, Jabatan Muzium.

1985 «Nisan Aceh Bertulis di Semenanjung Malaysia », Purba, 4, p. 6-22.

1990 Kota-Kota Melayu, Kuala Lumpur, Dewan Bahasa dan Pustaka.

ABDUl SAMAD BIN AHMAD (éd.)

1986 Sulalatus Salatin (Sejarah Melayu), Kuala Lumpur, Dewan Bahasa dan Pustaka, [1 ${ }^{\text {re }}$ éd., 1979].

AMBARY, Hasan Muarif

1984 L'art funéraire musulman en Indonésie des origines au XIX siècle. Étude épigraphique et typologique, thèse de doctorat, Paris, EHESS.

ANDAYA, Leonard Yuzon

1975 The Kingdom of Johore (1641-1728), Kuala Lumpur, Oxford University Press.

Cushman, Jennifer W. \& MiLner, Anthony Crothers

1979 «Eighteenth and nineteenth-century Chinese accounts of the Malay Peninsula », JMBRAS, 52/1, p. 1-56.

DAMAIS, Louis-Charles

1968 «L'épigraphie musulmane dans le sud-est asiatique », BEFEO, 54, p. 567604.

FOSTER, W. (éd.)

1995 The Voyage of Thomas Best to the East Indies (1612-1614), New Delhi/Madras, Asian Educational Services, [1 ${ }^{\text {re }}$ éd., 1934].

GrBSON-HILL, Carl Alexander

1955 «Johore Lama and other ancient sites on the Johore River », JMBRAS, 28/2, p. 126-198.

1956 "On the alleged death of Sultan Ala'u'd-din of Johore at Aceh, in 1613 », $J M B R A S, 29 / 1$, p. 125-145. 
GRoENEVELDT, Willem Pieter

1880 Notes on the Malay Archipelago and Malacca Compiled from Chinese Sources, Batavia (VBG, 39).

HAN WAI TOON

1948 "A Study on Johore Lama», Journal of the South Seas Society, 5/2, p. 1734.

HERVEY, Dudley Francis Amelius

1879 «A trip to Gunong Blumut », JSBRAS, 3, p. 85-115.

ISMAIL HUSSEIN (éd.)

1992 «Hikayat Negeri Johor - A Nineteenth Century Bugis History Relating Events in Riau and Selangor ", in R. O. Winstedt, 1992, p. 227-284.

KAMARUDIN AB. RAZAK

1998 Peninggalan Sejarah di Pesisiran Sungai Johor, Johor Bahru, Yayasan Warisan Johor.

KRATZ, Ernst Ulrich (éd.)

1973 Peringatan Sejarah Negeri Johor. Eine Malaiische Quelle zu Geschichte Johors im 18. Jahrhundert, Wiesbaden, Otto Harrassowitz.

LEUPE, P. A.

1936 "The Siege and Capture of Malacca from the Portuguese in 1640-1641. Translated by McHacobian from 'Berigten van het Historisch Genootschap te Utrecht', 1859, 128-429 », JMBRAS, 14/1, p. 1-178.

LIAW YOCK FANG (éd.)

1976 Undang-undang Melaka, The Laws of Melaka, Den Haag, M. Nijhoff (Bibliotheca Indonesica, 13).

LINEHAN, William

1973 A History of Pahang, Kuala Lumpur, Malaysian Branch of the Royal Asiatic Society, [1 ${ }^{\text {re }}$ éd., 1936].

LOMBARD, Denys

1967 Le sultanat d'Atjéh au temps d'Iskandar Muda, 1607-1636, Paris, EFEO (PEFEO, 61).

MARSDEN, William

1986 The History of Sumatra. Introduction by John Bastin, Kuala Lumpur, Oxford University Press, [ $1^{\text {re }}$ éd., 1783].

MAXWELL, William Edward

1882 «The History of Perak from Native Sources », JSBRAS, 9, p. 85-108.

MENDES PINTO, Fernâo

1991 Pérégrination. Traduit du portugais par R. Viale, Paris, La Différence, [1 ${ }^{\text {re }}$ éd., 1614].

MOHD. YUSOF MD. NOR (éd.)

1984 Salasilah Melayu dan Bugis, Petaling Jaya, Fajar Bakti.

MoOR, J. H.

1837 Notices of the Indian Archipelago and adjacent countries, Singapore.

NETSCHER, Elisa

1870 De Nederlanders in Djohor en Siak, 1602 tot 1865, historische beschrijving, Batavia, Bruining \& Wijt (VBG, 35). 
NORJIHAN NORDIN

1994 «Sejarah Kampung Panchor: Peranannya di Awal Kurun Ke-18 », in Abdullah Zakaria bin Ghazali \& Zainal Abidin Borhan (éd.), Johor dahulu dan sekarang, Kuala Lumpur, Persatuan Muzium Malaysia, p. 33-46.

NUR-UD-DIN AR-RANIRI, SYEIKH

1992 Bustan al-Salatin, Bab. II, Fasal 13, Siti Hawa Haji Salleh (éd.), Kuala Lumpur, Dewan Bahasa dan Pustaka, [1 ${ }^{\text {re }}$ éd., 1966].

OTHMAN BIN MOHD. YATIM

1985 Batu Aceh : a study of 15th-19th century Islamic gravestones in Peninsular Malaysia, PhD dissertation, University of Durham.

1988 Batu Aceh. Early Islamic Gravestones in Peninsular Malaysia, Kuala Lumpur, Muzium Negara.

OTHMAN BIN MOHD. YATIM \& ABDUl HALIM NASIR

1990 Epigrafi Islam Terawal di Nusantara, Kuala Lumpur, Dewan Bahasa dan Pustaka.

PERRET, Daniel, KAMARUdin AB. RAZAK et Ludvik Kalus (contribution épigraphique)

1999 Batu Aceh. Warisan Sejarah Johor, Johor Bahru, EFEO/Yayasan Warisan Johor (paru en nov. 2000).

RAJA ALI HAJI ibn AHMAD

1982 The Precious Gift. Tuhfat al-Nafis. An annotated translation by V. Matheson \& B. Watson Andaya, Kuala Lumpur, Oxford University Press.

1991 Tuhfat al-Nafis-Sejarah Melayu-Islam, V. Matheson-Hooker (éd.), Kuala Lumpur, Dewan Bahasa dan Pustaka.

RONKEL, Philippus Samuel van

1920 «Grafmonumenten op het maleische schiereiland in een oud maleisch werk vermeld », $B K I, 76$, p. 162-171.

SHAHAROM HUSAIN

1995 Sejarah Johor-Kaitannya Dengan Negeri Melayu, Kuala Lumpur, Penerbit Fajar Bakti.

SHELLABEAR, William Girdlestone (éd.)

1994 Sejarah Melayu, Kuala Lumpur, Fajar Bakti, [1 ${ }^{\text {rc }}$ éd., 1898].

SOUSA PINTO, Paulo Jorge Corino de

1994 Portugueses e Malaios - Malaca e os sultanatos de Johor e Achém 15751619, dissertação de mestrado, Lisboa, Faculdade de Ciências Sociais e Humanas.

SudjIMAN, PANUTI H. M. (éd.)

1983 Adat Raja-Raja Melayu, Jakarta, Penerbit Universitas Indonesia.

TERPSTRA, $\mathrm{H}$.

1938 De factorij der Oostindische Compagnie te Patani, 's Gravenhage, Martinus Nijhoff (Verhandelingen van het Koninklijk Instituut voor Taal-, Land- en Volkenkunde, 1).

TIELE, Pieter Anton

1886 «De Europeers in den Maleischen Archipel», BKI, 35, p. 257-355.

WALES, Horace Geoffrey Quaritch

1940 "Archaeological Researches on Ancient Colonization in Malaya », $J M B R A S, 18 / 1$, p. 1-85. 
WELD, FREDERIC A.

1887 Map of the Malay Peninsula, Gov. of Straits Set., Pub. under the auspices of the Straits Branch of the Royal Asiatic Society.

WINSTEDT, RICHARD OLAF

1932a «The Bendaharas and Temenggongs », JMBRAS, 10/1, p. 56-66.

1932b «A History of Johore (A.D. 1365-1895) », JMBRAS, 10/3, p. 1-158.

1992 A History of Johore (1365-1941). With a final chapter by Prof. Dato' Khoo Kay Kim, Kuala Lumpur, Malaysian Branch of the Royal Asiatic Society, reprint no. $6,\left[1^{\text {re }}\right.$ éd. 1979$]$. 